\title{
Organisierte Zivilgesellschaft
}

Jana Priemer Ziviz | Antje Bischoff Bundesverband Deutscher Stiftungen | Christian Hohendanner IAB | Ralf Krebstakies Bundesverband Deutscher Stiftungen | Boris Rump DOSB | Wolfgang Schmitt BAGFW

Warum benötigen wir empirische Daten zu Vereinen, Stiftungen und anderen gemeinnützigen Organisationen? Der Großteil bürgerschaftlichen Engagements findet nach wie vor überwiegend in Vereinen und anderen Organisationen des Dritten Sektors statt. Sie bilden also einen organisationalen Rahmen für bürgerschaftliches Engagement (vgl. Abschnitt 4.8). Zudem leisten diese Organisationen vielfältige gesellschaftliche Beiträge. Sie ergänzen die von Staat und Wirtschaft erbrachten Angebote und Leistungen vor allem als Dienstleister für zentrale Bereiche der sozialen Daseinsvorsorge, wie Kinderbetreuung und Altenpflege. Als Träger zahlreicher sozialer Einrichtungen sind sie auch bedeutende Arbeitgeber (vgl. Kapitel 5). Vereine und andere Organisationen sind wichtige Bildungsträger, nicht nur als Einrichtungsträger von Kindertagesstätten und Schulen oder Hochschulen in privater Trägerschaft. Sie ergänzen als Anbieter von Nachmittagsbetreuungsangeboten das schulische Angebot, daneben bieten sie eine breite Palette außerschulischer Bildungsangebote an, oftmals in "ganz normalen" Vereinen wie Sport-, Kultur- oder Umweltvereinen. Ohne Vereine gäbe es viele Sport-, Kulturund Freizeitangebote gar nicht. In strukturschwachen Regionen ergänzen Vereine, Stiftungen, gemeinnützige GmbHs und Genossenschaften immer häufiger auch als Träger von Bürgerbussen, Dorfläden oder Dorfkneipen die öffentlichen Versorgungsangebote. In akuten Bedarfssituationen, etwa bei Flutkatastrophen, sind es oftmals die Organisationen der Zivilgesellschaft, die schnell und unbürokratisch helfen. Auch in der Flüchtlingshilfe haben sich, neben den vielen Einzelpersonen und informellen Initiativen, viele Organisationen der Zivilgesellschaft eingebracht. Durch ihr vielfältiges Angebot leisten diese Organisationen damit wichtige Beiträge zur gesellschaftlichen Integration. 


\subsection{Konzeptionelle Einordnung ${ }^{1}$}

Was meinen wir überhaupt, wenn wir von organisierter Zivilgesellschaft sprechen? Eine einheitliche Definition von Zivilgesellschaft gibt es nicht. Die inhaltliche Ausgestaltung des Konzepts Zivilgesellschaft fällt je nach Forschungsdisziplin unterschiedlich aus. Vorherrschend sind normativ geprägte Ansätze, die Zivilgesellschaft mit einem demokratischen Gemeinwesen und einer zivilen, also "guten“ und gerechten, Gesellschaft, gleichsetzen. Für die folgenden Ausführungen sind zudem akteurszentrierte Ansätze relevant. Zivilgesellschaftlich handelnde Einzelpersonen können sich zu lose bzw. informell gestalteten Initiativen oder zu hoch formalisierten Organisationen zusammenschließen. Beispielhaft angeführt werden für solche Zusammenschlüsse meist Vereine (sowohl eingetragene als auch nicht eingetragene) und Stiftungen. Im internationalen Kontext ist die Rede von NGOs und NPOs, also Non-Governmental beziehungsweise Non-Profit-Organisationen. Letztere verweisen bereits über den Begriff "non profit“ in ihrer Namensgebung auf Kriterien, die diese Organisationen laut dem international verbreiteten Dritter-Sektor-Ansatz zu erfüllen haben, um dem Dritten Sektor zugeordnet zu werden - also jenem Bereich, der weder Staat noch Markt noch Familie ist. Er umfasst formal organisierte, private, selbstverwaltete und von Freiwilligkeit gekennzeichnete Organisationen, die ihre durch ehrenamtliche Leistungen erzielten Gewinne nicht an die Mitglieder oder Eigentümer ausschütten, sondern investieren. Damit weist der Dritte Sektor hinreichend Bezüge zur Zivilgesellschaft auf, um ihn als konzeptionellen Unterbau für eine Analyse der Organisationen der Zivilgesellschaft heranzuziehen (Anheier et al. 2000, S. 75).

Der Dritter-Sektor-Ansatz hat zwar einen Organisationsbezug, ist jedoch im deutschen Kontext für den empirischen Zugang nur bedingt geeignet, da die Grenzen mitunter fließend sind und eine eindeutige Zuordnung zu den Sektoren Staat, Markt und Zivilgesellschaft daher nicht ohne weiteres möglich ist (vgl. Krimmer und Priemer 2013, S. 8 ff., S. 78 ff.; Priemer et al. 2017, S. 50 ff.). Schwierigkeiten bei der Zuordnung ergeben sich beispielsweise bei Unternehmensstiftungen, deren Vermögen aus den wirtschaftlichen Aktivitäten eines Unternehmens stammt. Staatliche Ausgründungen, die als eingetragene Vereine agieren, verursachen ebenfalls Schwierigkeiten bei der Zuordnung. Laut Dritter-SektorAnsatz wären sie der staatlichen Sphäre zuzurechnen, da ihre Gremien überwiegend mit öffentlichen Amtsinhabern besetzt sind und sie auch überwiegend durch öffentliche Mittel finanziert werden. Als Beispiel können die kommunalen Spitzenverbände angeführt werden, bei denen es sich um Zusammenschlüsse von

1 Dieses Unterkapitel wurde verfasst von Jana Priemer, ZiviZ. 
kommunalen Gebietskörperschaften handelt, von denen einige die Rechtsform des eingetragenen Vereins haben.

Aus forschungspragmatischen Gründen hat sich der Zugang über die Rechtsformen bewährt. Das sind neben eingetragenen Vereinen und Stiftungen auch andere gemeinnützige Organisationsformen wie gemeinnützige Gesellschaften mit beschränkter Haftung ( $\mathrm{gGmbH}$ ) oder gemeinnützige Genossenschaften sowie gemeinnützige Aktiengesellschaften und neuerdings auch gemeinnützige Unternehmergesellschaften. Diese lassen sich beispielsweise über öffentlich zugängliche Register identifizieren und abgrenzen. Ein Zugang über diese Rechtsformen hat Bezüge sowohl zum Dritte-Sektor-Konzept als auch zum akteurszentrierten Ansatz der Zivilgesellschaft, weshalb im Folgenden von der organisierten Zivilgesellschaft gesprochen wird, obgleich auch hierbei Unschärfe und Einschränkungen hinzunehmen sind, etwa hinsichtlich des im Zivilgesellschaftskonzept verankerten normativen Anspruchs der Zivilität. Denn längst nicht alle dieser genannten Organisationen sind immer „zivil“ oder „zivilgesellschaftlich“ (Roth 2003). Informelle Zusammenschlüsse können bei diesem Zugang nicht berücksichtigt werden, auch wenn diese gerade in der jüngeren Vergangenheit an Bedeutung gewonnen haben (vgl. Abschnitt 4.8). Insbesondere das Engagement in der Flüchtlingshilfe in den Jahren 2015 und 2016 zeichnete sich zu erheblichen Teilen durch spontan entstandene Initiativen oder Projekte und andere selbstorganisierte Gruppen aus (Karkayali und Kleist 2017; Mutz et al. 2015). Der spontane Charakter solcher informellen Zusammenschlüsse und deren Kurzlebigkeit stellen besondere Herausforderungen an einen empirischen Zugang (Skurnog 2017, S. 8 ff.; BMFSFJ 2017, S. 9), so dass diese Organisationsformen bislang kaum in Forschungsvorhaben berücksichtigt werden (konnten).

\subsection{Größe und Entwicklung der organisierten Zivilgesellschaft in Deutschland ${ }^{2}$}

Die organisierte Zivilgesellschaft wächst. Die Zahlen der eingetragenen Vereine, Stiftungen, gemeinnützigen $\mathrm{GmbHs}$ und Genossenschaften steigen seit einigen Jahren stetig. Obwohl sich jedes Jahr viele Organisationen auch wieder auflösen, ist die Bilanz nach wie vor positiv. So wurden im Jahr 2013 zwar 10512 Vereine aus den Vereinsregistern gelöscht, doch auch 15084 neue eingetragen (Bundesamt für Justiz 2017).

Ein deutliches Wachstum hat es in den vergangenen Jahren vor allem bei den Stiftungen und bei den gemeinnützigen GmbHs gegeben. Der Stiftungsbestand

2 Dieses Unterkapitel wurde verfasst von Jana Priemer, ZiviZ. 
Tabelle 3.1 Entwicklung von Vereinen, Stiftungen, gGmbHs und Genossenschaften im Zeitverlauf

\begin{tabular}{|c|c|c|c|c|}
\hline Jahr & Vereine* $^{*}$ & Stiftungen ${ }^{* *}$ & gGmbHs*** & Genossenschaften* \\
\hline 1995 & 416861 & & & 13068 \\
\hline 1996 & 433149 & & & 13974 \\
\hline 1997 & 449104 & & & 13661 \\
\hline 1998 & 464427 & & & 13805 \\
\hline 1999 & 477860 & & & 13459 \\
\hline 2000 & 490875 & & & 13086 \\
\hline 2001 & 503682 & 10503 & & 12706 \\
\hline 2002 & 515283 & 11277 & & 12342 \\
\hline 2003 & 523529 & 12000 & & 12147 \\
\hline 2004 & 535832 & 12670 & & 12061 \\
\hline 2005 & 548517 & 13490 & & 11619 \\
\hline 2006 & 554097 & 14401 & & 10736 \\
\hline 2007 & 559607 & 15449 & 16400 & 9583 \\
\hline 2008 & 566171 & 16406 & 14100 & 9593 \\
\hline 2009 & 570374 & 17372 & 15100 & 9614 \\
\hline 2010 & 576357 & 18162 & 17300 & 9134 \\
\hline 2011 & 582339 & 18946 & 20000 & 9283 \\
\hline 2012 & 588368 & 19551 & 20500 & 8561 \\
\hline 2013 & 591759 & 20150 & 22600 & 8719 \\
\hline 2014 & 630143 & 20784 & 22100 & 8729 \\
\hline 2015 & 598210 & 21301 & 25200 & 8768 \\
\hline 2016 & 602602 & 21806 & 25300 & 8743 \\
\hline
\end{tabular}

Quellen: *Bundesamt für Justiz 2017; ** rechtsfähige Stiftungen des bürgerlichen Rechts (Bundesverband Deutscher Stiftungen: Umfrage unter den Stiftungsaufsichtsbehörden, Stichtag jeweils 31. Dezember. *** IAB-Betriebspanel 2007-2016, hochgerechnete Werte, gGmbH: Betriebe mit der Rechtsform GmbH, die zugleich im Sinne des Steuerrechts als gemeinnützige, mildtätige oder kirchliche Einrichtung steuerbegünstigt sind. 
hat sich seit dem Jahr 2000 annähernd verdreifacht. Die Datenbank des Bundesverbandes Deutscher Stiftungen zählt heute (2018) mehr als 30 ooo Stiftungen. Die Anzahl der als gemeinnützig eingestuften GmbHs hat sich nach Angaben des IAB-Betriebspanels in einem Zeitraum von zehn Jahren (2007 bis 2016) von rund 16 ooo auf gut 25000 Einrichtungen ebenfalls deutlich erhöht, wenn auch nicht so drastisch wie die der Stiftungen.

Zudem gewinnen weitere Rechtsformen wie die gemeinnützige Aktiengesellschaft und die gemeinnützige Unternehmergesellschaft an Popularität, wenngleich diese quantitativ noch eine untergeordnete Rolle spielen. Beide Rechtsformen machen an der Gesamtzahl der Organisationen der Zivilgesellschaft bislang nicht einmal 1 Prozent aus.

Eingetragene Vereine, Stiftungen gemeinnützige GmbHs und Genossenschaften sind zwar alle der organisierten Zivilgesellschaft zuzuordnen, doch sie weisen mitunter sehr spezifische Charakteristika auf, so dass im Folgenden eingetragene Vereine, Stiftungen und wirtschaftlich aktive Organisationen, zu denen vor allem gemeinnützige GmbHs und Genossenschaften, jedoch auch ein Teil der Stiftungen und der eingetragenen Vereine zählen, separat betrachtet werden. Dabei werden unterschiedliche Datenquellen herangezogen. Die Darstellung der eingetragenen Vereine basiert auf den Daten des ZiviZ-Surveys. Als Prototyp der deutschen Vereinslandschaft wird der Sport gesondert dargestellt; dafür wird auf die Daten der Sportentwicklungsberichte zurückgegriffen. Abschließend werden wirtschaftlich aktive Organisationen, basierend auf den Daten des IAB-Betriebspanels und des Statistischen Bundesamtes, dargestellt. Ergänzt wird diese Darstellung durch Daten zur Wohlfahrtspflege von der Bundesarbeitsgemeinschaft der Freien Wohlfahrtspflege (BAGFW). 


\section{Herausforderung bei der Bestandsaufnahme von Organisationen der Zivilgesellschaft}

Derzeit gibt es noch erhebliche Unsicherheiten hinsichtlich der Bestände von Vereinen, Stiftungen und anderen Organisationen. Im Gegensatz zu anderen Ländern gibt es in Deutschland keine einheitlichen Registrierungspflichten, auch wenn mit dem 2017 eingerichteten Transparenzregister ein erster Schritt in diese Richtung gegangen wurde.

Das gemeinsame Registerportal der Länder (www.registerportal.de) und das Verzeichnis Deutscher Stiftungen sind zurzeit die wichtigsten Quellen zur Ermittlung der Bestände der Organisationen.

Über das gemeinsame Registerportal der Länder kann die Zahl der eingetragenen Vereine ermittelt werden. Im Registerportal sind neben dem Vereinsnamen für jedes Registergericht die Unternehmensträgerdaten zugänglich. Dort sind zum Teil auch die Kontaktdaten hinterlegt. Als problematisch erweist sich für den Forschungszugang, dass die Vereinsregister nicht immer aktuell sind. Nicht alle Vereine teilen Änderungen mit, zum Teil auch, weil Änderungen im Vereinsregister mit Kosten verbunden sind. Einige Vereine werden nie aus den Registern gelöscht, obwohl sie längst nicht mehr aktiv sind. Wie hoch der Anteil der nicht mehr aktiven, aber in den Vereinsregistern geführten Vereine in der Realität ist, ist schwer abzuschätzen, weshalb die Zahl von mehr als 600 ooo eingetragenen Vereinen allenfalls ein Näherungswert sein kann, wenngleich ein hilfreicher, da er eine erste Orientierung zur Zahl der Vereine in Deutschland bietet.

Die meisten der heute tätigen Stiftungen sind im Verzeichnis Deutscher Stiftungen des Bundesverbandes Deutscher Stiftungen aufgeführt. Doch die Erfassung des Stiftungsbestandes ist ebenfalls schwierig. Da der Begriff der Stiftung im Gesetz nicht definiert ist, existiert neben den klassischen rechtsfähigen Stiftungen des bürgerlichen Rechts eine Vielzahl von Stiftungen anderer Rechtsformen, wie zum Beispiel Stiftungs-GmbHs, Stiftungsvereine, nicht rechtsfähige Stiftungen oder Stiftungen der öffentlichen Hand. Nur die rechtsfähigen Stiftungen des bürgerlichen Rechts unterstehen der staatlichen Stiftungsaufsicht, so dass ausschließlich für diese Rechtsform die genaue Anzahl bekannt ist. Informationen zu den Stiftungen veröffentlichen die Aufsichtsbehörden in ihren Online-Stiftungsverzeichnissen. Zu Stiftungs-GmbHs und -vereinen finden sich Daten im Registerportal der Länder (s. o.), während für nicht rechtsfähige Stiftungen oder öffentlich-rechtliche Stiftungen keine zentralen Quellen vorliegen. Einige evangelische Landeskirchen veröffentlichen von ihnen beaufsichtigte kirchliche Stiftungen in einer Onlinedatenbank (www.stiftungsdatenbank.de/register-kirchliche-stiftungen). Das neu eingerichtete Transparenzregister schafft diesbezüglich kaum Abhilfe, da nur ein Teil der nicht rechtsfähigen Stiftungen verpflichtet ist, sich hier einzutragen.

Während Genossenschaften im Handelsregister (ebenfalls über das Registerportal) abgerufen werden können, ist es weitaus schwieriger, die Bestände der gemeinnützigen GmbHs zu ermitteln. GmbHs können zwar grundsätzlich auch im Handelsregister nachgeschlagen werden. Die Herausforderung besteht jedoch darin, dass nicht jede als gemeinnützig anerkannte $\mathrm{GmbH}$ das "gemeinnützig“ im Namen führt, so dass sich nur schwer ermitteln lässt, wie viele der $683000 \mathrm{GmbHs}$ (IAB-Betriebspanel 2016) gemeinnützig sind. Einen Zugang bietet das IAB-Betriebspanel. Seit 2007 wird dort erfasst, ob die befragten Betriebe und Einrichtungen im Sinne des Steuerrechts als gemeinnützige, mildtätige oder kirchliche Einrichtung steuerbegünstigt sind. Zudem wird gesondert die Rechtsform der Organisation erfasst. Die Kombination beider Merkmale ermöglicht es, gemeinnützige Organisationen mit unterschiedlichen Rechtsformen hochzurechnen. 


\subsection{Eingetragene Vereine ${ }^{3}$}

Den Verein gibt es nicht. Die rund 600 ooo eingetragenen Vereine in Deutschland zeichnen sich durch eine extrem hohe Heterogenität aus. Es gibt kaum ein Anliegen oder ein Interesse, das nicht von mindestens einem Verein vertreten wird. Vereine unterscheiden sich nicht nur in ihren Aktivitäten, sondern auch in ihren Strukturmerkmalen, zum Beispiel hinsichtlich ihrer Größe oder ihrer Finanzierungsstrukturen. Solche Unterschiede lassen sich etwa zwischen einzelnen Handlungsfeldern feststellen - zum Beispiel zwischen Sportvereinen und Vereinen, die im Handlungsfeld Bürger- und Verbraucherinteressen aktiv sind. Über die Unterschiede gibt der ZiviZ-Survey einen guten Überblick. Der ZiviZ-Survey ist derzeit die einzige repräsentative Befragung zivilgesellschaftlicher Organisationen in Deutschland. Er untersucht eingetragene Vereine, Stiftungen, Genossenschaften und gemeinnützige GmbHs. Am ZiviZ-Survey 2017 haben sich 6334 Organisationen beteiligt. Die Erhebung wurde von September 2016 bis Februar 2017 durchgeführt (Priemer et al. 2017, S. 50 ff.). Da der ZiviZ-Survey aus allen Organisationen der Zivilgesellschaft eine repräsentative Stichprobe zieht und erst nachträglich durch die Vereine selbst, im Rahmen der Erhebung, eine Zuordnung zu den Handlungsfeldern erfolgt, können Unterschiede zwischen einzelnen Organisationssegmenten sowie Veränderungen und Verschiebungen aufgezeigt werden.

Zwei zentrale Merkmale, auf die im Folgenden besonders eingegangen wird, sind die Mitgliederzahlen und die Zahl der freiwillig Engagierten. Diese liefern nicht nur Informationen zur Größe der Vereine, sondern lassen auch Schlüsse zu personellem und finanziellem Ressourcenpotenzial zu. Denn Vereine finanzieren sich meist zu erheblichen Teilen über ihre Mitgliedsbeiträge und haben in der Regel wenig finanziellen Spielraum (vgl. Kapitel 6), so dass die meisten Aktivitäten ausschließlich über ehrenamtliches Engagement getragen werden.

Räumliche Verteilung: Neben den Daten des ZiviZ-Surveys kann die Vereinsliste 2016 für eine Analyse der räumlichen Verteilung der Vereine in Deutschland herangezogen werden. Abgerufen wurden die Daten über das Onlineportal des Handelsregisters, das gemeinsame Registerportal der Länder. Etwa 98 Prozent der eingetragenen Vereine, die im August 2016 in den Vereinsregistern geführt wurden, sind in dieser Liste enthalten. Auf Basis der Vereinsregisterauswertungen kommen wir für das Jahr 2016 auf einen Vereinsbestand von 603 886. Das ist eine Differenz von knapp 1300 Vereinen zu den Zahlen des Bundesamts für Justiz (2017), was einer Abweichung von 0,2 Prozent entspricht. Da das Bundesamt für Justiz

3 Dieses Unterkapitel wurde verfasst von Jana Priemer, ZiviZ. 
keine Angaben auf Bundesländerebene macht, wird hierzu die Vereinsliste 2016 herangezogen. $\mathrm{Zu}$ berücksichtigen sind bei der Interpretation der Daten die bereits weiter oben thematisierten Unsicherheiten. Eine weitere Einschränkung folgt aus den nicht vorhandenen Informationen zur Zahl der Mitglieder und Engagierten in den Vereinen. Wir wissen daher nicht, ob die Vereine in einer bestimmten Region eher groß oder eher klein sind. Ein großer Verein mit mehreren Hundert Mitgliedern und Engagierten kann mitunter ebenso viele Personen binden wie viele kleine Vereine. Somit sind Aussagen zur sozialen Integrationskraft über die reine Zahl der Vereine nur bedingt möglich.

Im Durchschnitt kommen auf 1 ooo Einwohner etwa sieben eingetragene Vereine. Die Vereine sind jedoch nicht gleichmäßig über Deutschland verteilt. Die Vereinsdichte variiert zwischen sechs Vereinen je 1 ooo Einwohner in Hamburg und zehn im Saarland. Tendenziell gibt es, bezogen auf die Einwohnerzahl, in den neuen Bundesländern mehr Vereine als in den alten, wobei natürlich Unterschiede zwischen den Bundesländern bestehen. In Brandenburg und Sachsen etwa gibt es auf 10 ooo Einwohner sieben Vereine, in Sachsen-Anhalt und Thüringen neun. Auch innerhalb der Bundesländer gibt es regionale Unterschiede, etwa zwischen strukturstarken und strukturschwachen Regionen oder zwischen städtischem und ländlichem Raum. Weitere Analysen auf Basis der Vereinsliste, unter anderem von Zusammenhängen zwischen verschiedenen sozial-räumlichen Parametern und der Vereinsdichte, sind zeitnah geplant.

Abbildung 3.1 Vereine nach Siedlungsgröße (in Prozent)

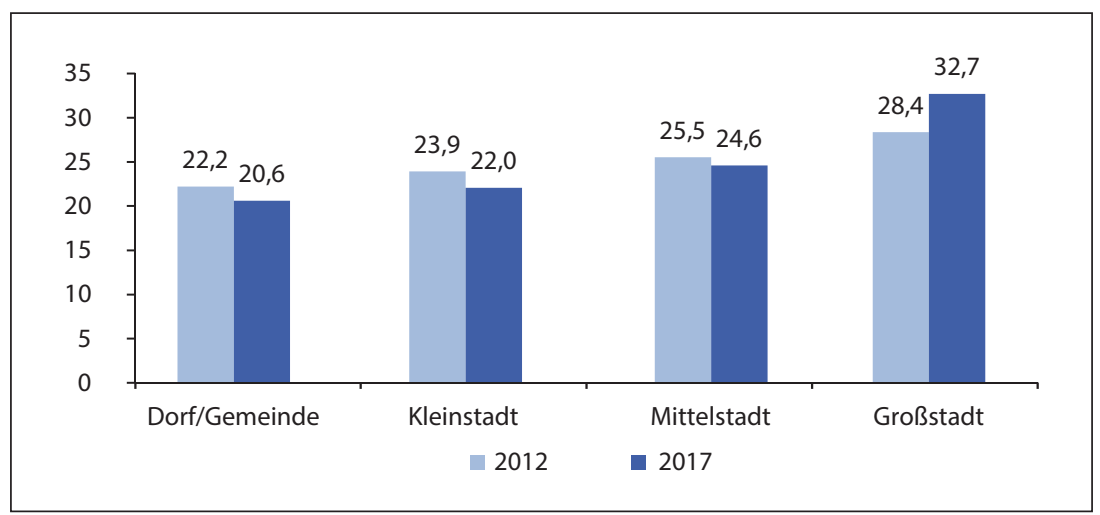

Quelle: ZiviZ-Survey 2017, nur Vereine, $n=6461$ (gewichtet), davon fehlend: 90; ZiviZ-Survey 2012, nur Vereine, $n=3604$ (gewichtet), davon fehlend: 68 . 
Tabelle 3.2 Vereine nach Bundesland im August 2016

\begin{tabular}{lccc}
\hline & Anzahl & Prozent & je 1 000 EW \\
\hline Baden-Württemberg & 83562 & 13,8 & 7,7 \\
Bayern & 90796 & 15,0 & 7,1 \\
Berlin & 24643 & 4,1 & 7,0 \\
Brandenburg & 17924 & 3,0 & 7,2 \\
Bremen & 3727 & 0,6 & 5,6 \\
Hamburg & 9788 & 1,6 & 5,5 \\
Hessen & 50283 & 8,3 & 8,1 \\
Mecklenburg-Vorpommern* & 12700 & 2,1 & 7,9 \\
Niedersachsen & 56685 & 9,4 & 7,2 \\
Nordrhein-Westfalen & 120207 & 19,9 & 6,7 \\
Rheinland-Pfalz & 37989 & 6,3 & 9,4 \\
Saarland & 10337 & 1,7 & 10,4 \\
Sachsen & 29777 & 4,9 & 7,3 \\
Sachsen-Anhalt & 19142 & 3,2 & 8,5 \\
Schleswig-Holstein & 17229 & 2,9 & 6,0 \\
Thüringen & 19097 & 3,2 & 8,8 \\
\hline Deutschland & 603886 & 100,0 & 7,3 \\
\hline
\end{tabular}

Quelle: Vereinsliste 2016. Die Daten basieren auf den Angaben der Vereinsregister, die im Gemeinsamen Registerportal der Länder digital zugänglich sind. Stand der Daten ist August 2016.

* Über das Registerportal konnten für Mecklenburg-Vorpommern nur 5923 Vereine abgerufen werden. Auf Basis der Zahlen des Bundesamtes für Justiz von 2014 schätzten wir den Vereinsbestand auf 12770.

Auch die Daten des ZiviZ-Surveys belegen eine ungleiche Verteilung der Vereine im Raum. Analog zur Bevölkerung, die überwiegend in Städten lebt, sind auch die meisten Vereine in Städten ansässig. Da Vereine in der Regel von der lokalen Bevölkerung getragen werden, wirkt sich die zunehmende Verstädterung auch auf die Vereinslandschaft aus. Heute sind 42,6 Prozent der Vereine in Kleinstädten oder in kleineren Gemeinden verortet, 2012 waren es noch 46,1 Prozent. Der Anteil der in Großstädten ansässigen Vereine ist hingegen um 5 Prozentpunkte gestiegen, so dass heute jeder dritte Verein (32,7 Prozent) in einer Großstadt mit mindestens 100 ooo Einwohnern zu finden ist. Es ist zwar bislang nur eine leich- 
te Verschiebung, die jedoch dürfte sich vor dem Hintergrund der zunehmenden Alterung der Landbevölkerung durch Abwanderung vor allem junger Menschen (Kröhnert et al. 2011) weiter verschärfen. Erste Hinweise in diese Richtung liefern auch die Befunde zur Mitgliederentwicklung in Vereinen des ländlichen Raums (vgl. Tab. 3.8).

Handlungsfelder: Viele Vereine (42,7 Prozent) betätigen sich in mindestens zwei Handlungsfeldern ${ }^{4}$. Der lokale Umweltverein etwa betreibt Umweltbildung vor Ort und ordnet sich nicht nur dem Umwelt- und Naturschutz zu, sondern auch dem Bereich Bildung 5 . Dominiert wird das Vereinswesen in Deutschland nach wie vor von Sport- und Kulturvereinen, Freizeit- und Geselligkeitsvereinen sowie von den Vereinen der Handlungsfelder Bildung und Soziale Dienste. Sie machen zusammen fast zwei Drittel der Vereinslandschaft aus. Davon ist Sport noch immer das größte Handlungsfeld, obwohl es zwischen 2012 und 2017 leichte Verschiebungen zwischen den drei größten Handlungsfeldern gegeben hat. Im Handlungsfeld Bildung gab es in den vergangenen Jahren zahlreiche Neugründungen, so dass Bildung auf Platz zwei aufgerückt ist und zumindest die relativen Anteile der Sport- und Kulturvereine rückläufig sind.

Bildung hat sich erst in den vergangenen 20 Jahren zu einem relevanten Aktivitätsfeld unter den Vereinen entwickelt, weshalb es sich um ein vergleichsweise junges Handlungsfeld handelt. Etwa jeder achte der heute bestehenden Bildungsvereine (12,9 Prozent) wurde seit 2012 gegründet, also innerhalb der vergangenen 6 Jahre. Zu den freizeitbezogenen Vereinen hingegen gehören größtenteils Traditionsvereine, die es bereits lange gibt. Jeweils rund ein Drittel der Sport- (34,3 Prozent) und Freizeitvereine (30,1 Prozent) existierten in ihren Ursprüngen bereits

4 Die Organisationen wurden gebeten, ihre Aktivitäten Handlungsfeldern zuzuordnen. Es standen 14 Kategorien zur Auswahl, darunter Kultur und Medien, Sport, Freizeit und Geselligkeit, Gesundheitswesen, Soziale Dienste, Bildung und Erziehung. In der organisierten Zivilgesellschaft ist es üblich, dass eine Organisation Aktivitäten in mehreren Handlungsfeldern ausübt. Zur Veranschaulichung ist der Deutsche Alpenverein (DAV) mit seinen zahlreichen Landesverbänden und Ortsgruppen zu nennen. Der DAV versteht sich sowohl als Sport- als auch als Naturschutzverein. Annähernd die Hälfte aller Organisationen (43 Prozent) hat mehr als ein Handlungsfeld angegeben. Oftmals sind die Grenzen zwischen den Handlungsfeldern auch fließend, zum Beispiel zwischen sozialen Diensten und Gesundheit, etwa bei Einrichtungen, die auch Pflegedienste anbieten. Um handlungsfeldspezifische Auswertungen vornehmen zu können, wurden die Organisationen dennoch gebeten, sich für das Handlungsfeld zu entscheiden, in dem sie überwiegend aktiv sind. Fast alle Organisationen (97 Prozent) konnten auf diese Weise einem Haupthandlungsfeld zugeordnet werden. Wenn nicht anders ausgewiesen, beziehen sich Angaben zu den Handlungsfeldern (gelegentlich sprechen wir auch von Bereichen) auf das Haupthandlungsfeld.

5 Das Handlungsfeld Bildung umfasst auch Erziehung. Der besseren Lesbarkeit wegen wird verkürzt Bildung verwendet. Gemeint ist immer das Handlungsfeld Bildung und Erziehung. 
Tabelle 3.3 Handlungsfelder der Vereine (in Prozent)

\begin{tabular}{lc}
\hline Sport & 22,6 \\
Bildung/Erziehung & 18,3 \\
Kultur/Medien & 16,1 \\
Freizeit/Geselligkeit & 7,9 \\
Soziale Dienste & 7,1 \\
Sonstiges & 4,3 \\
Kirchen/religiöse Vereinigungen & 4,0 \\
Gesundheitswesen & 3,5 \\
Umwelt-/Naturschutz & 3,1 \\
Bevölkerungs-/Katastrophenschutz & 3,1 \\
Internationale Solidarität & 2,9 \\
Bürger-/Verbraucherinteressen & 2,7 \\
Wissenschaft/Forschung & 2,3 \\
Wirtschafts-/Berufsverbände & 1,6 \\
Gemeinschaftliche Versorgungsaufgaben & 0,7 \\
\hline Gesamt & 100,0 \\
\hline
\end{tabular}

Quelle: ZiviZ-Survey 2017, nur Vereine, $\mathrm{n}=6461$ (gewichtet), davon fehlend: 162.

vor 1945 oder wurden in den ersten Jahren nach dem Zweiten Weltkrieg, bis einschließlich 1950, gegründet. Nur 5,1 Prozent der Vereine des Handlungsfeldes Bildung stammen aus jener Zeit. Viele neue Vereine wurden seit 2012 auch in den Handlungsfeldern Internationale Solidarität (22,2 Prozent), Bürger- und Verbraucherinteressen (21,3 Prozent) sowie Umwelt- und Naturschutz (18,o Prozent) gegründet.

Mitglieder: Die deutsche Vereinslandschaft ist überwiegend (60,9 Prozent) von kleinen Vereinen mit maximal 1oo Mitgliedern geprägt. Das sind oftmals auf lokaler Ebene agierende Vereine mit zum Teil wenigen Engagierten und meist nur geringen finanziellen Ressourcen. Es gibt auch große Vereine, die meist als Verbände agieren mehrere Tausend Mitglieder und viele Freiwillige haben, überregional aktiv sind und die Interessen vieler Menschen bündeln. Letztere sind, quantitativ betrachtet, eher Ausnahmen, auch wenn die öffentliche Wahrnehmung meist eine andere ist. Nur 15,1 Prozent der Vereine haben mehr als 300 Mitglieder; mehr als 
Tabelle 3.4 Gründungsjahre nach Handlungsfeld (in Prozent)

\begin{tabular}{lccll}
\hline & bis 1950 & $50 / 60 \mathrm{er}$ & $70 / 80 \mathrm{er}$ & seit 90ern \\
\hline Gemeinschaftliche Versorgungsaufgaben & 5,4 & 8,1 & 13,5 & 73,0 \\
Internationale Solidarität & 3,4 & 5,1 & 20,2 & 71,3 \\
Bildung/Erziehung & 5,1 & 5,6 & 22,7 & 66,6 \\
Sonstiges & 8,1 & 9,3 & 18,5 & 64,1 \\
Bürger-/Verbraucherinteressen & 18,3 & 7,3 & 11,6 & 62,8 \\
Gesundheitswesen & 8,3 & 3,7 & 25,8 & 62,2 \\
Soziale Dienste & 6,3 & 6,8 & 28,7 & 58,2 \\
Kirchen/religiöse Vereinigungen & 17,2 & 4,4 & 20,4 & 58,0 \\
Umwelt-/Naturschutz & 26,7 & 9,9 & 11,0 & 52,4 \\
Kultur/Medien & 21,0 & 8,0 & 20,9 & 50,1 \\
\hline Gesamt & 19,9 & 9,5 & 21,4 & 49,2 \\
\hline Wissenschaft/Forschung & 17,9 & 13,6 & 23,6 & 45,0 \\
Wirtschafts-/Berufsverbände & 19,4 & 10,8 & 30,1 & 39,8 \\
Freizeit/Geselligkeit & 30,1 & 11,5 & 24,1 & 34,3 \\
Bevölkerungs-/Katastrophenschutz & 48,9 & 6,9 & 11,7 & 32,4 \\
Sport & 34,3 & 16,4 & 20,9 & 28,3 \\
\hline
\end{tabular}

Quelle: ZiviZ-Survey 2017, nur Vereine, $n=6461$ (gewichtet), davon fehlend: 346 .

500 Mitglieder haben nur noch 5,6 Prozent der Vereine. Verbände machen weniger als ein Zehntel aller Vereine (8,4 Prozent) aus. Die meisten mitgliederstarken Vereine gibt es im Sport. Fast jeder dritte Sportverein (30,9 Prozent) hat mehr als 300 Mitglieder. So viele große Vereine gibt es in keinem anderen Handlungsfeld. Demgegenüber stehen zum Beispiel Internationale Solidarität und Bildung - zwei junge Handlungsfelder, von denen die überwiegende Mehrheit aus kleinen Vereinen (82,3 Prozent bzw. 77,6 Prozent) mit 100 oder weniger Mitgliedern besteht.

Die Entwicklung der Mitgliederzahlen der Vereine zeigt, dass der Verein mitnichten am Aussterben ist. Denn obwohl die Zahl der Vereine seit Jahren stetig ansteigt, sind die Mitgliederzahlen pro Verein (42,5 Prozent) stabil geblieben. Jeder dritte Verein (35,2 Prozent) berichtet sogar, dass er heute mehr Mitglieder hat als noch im Jahr 2012. Rückläufige Mitgliederzahlen vermelden „nur“ 22,3 Prozent. Das ist zwar ein ernst zu nehmender Wert, er legt jedoch noch nicht den 
Tabelle 3.5 Vereinsgröße nach Handlungsfeld (in Prozent)

\begin{tabular}{llll}
\hline & $\begin{array}{l}\text { klein } \\
\text { (bis 100 Mit- } \\
\text { glieder) }\end{array}$ & $\begin{array}{l}\text { mittelgroß } \\
\text { (101 bis 300 Mit- } \\
\text { glieder) }\end{array}$ & $\begin{array}{l}\text { groß } \\
\text { (mehr als } \\
\text { 300 Mitglieder) }\end{array}$ \\
\hline Sport & 40,4 & 28,6 & 30,9 \\
Wirtschafts-/Berufsverbände & 58,4 & 16,9 & 24,7 \\
Wissenschaft/Forschung & 52,2 & 26,1 & 21,6 \\
Bürger-/Verbraucherinteressen & 55,9 & 24,8 & 19,3 \\
Umwelt-/Naturschutz & 54,0 & 27,0 & 19,0 \\
\hline Gesamt & 60,9 & 24,0 & 15,1 \\
\hline Gesundheitswesen & 65,0 & 20,0 & 15,0 \\
Bevölkerungs-/Katastrophenschutz & 56,4 & 30,3 & 13,3 \\
Kirchen/religiöse Vereinigungen & 65,1 & 22,9 & 11,9 \\
Gemeinschaftliche Versorgungsaufgaben & 71,8 & 17,9 & 10,3 \\
Kultur/Medien & 65,6 & 25,2 & 9,3 \\
Freizeit/Geselligkeit & 62,1 & 28,7 & 9,2 \\
Sonstiges & 71,3 & 20,6 & 8,1 \\
Soziale Dienste & 66,7 & 25,5 & 7,8 \\
Bildung/Erziehung & 77,6 & 16,0 & 6,4 \\
Internationale Solidarität & 82,7 & 13,3 & 4,0 \\
\hline
\end{tabular}

Quelle: ZiviZ-Survey 2017, nur Vereine, $\mathrm{n}=6461$ (gewichtet), davon fehlend: 700.

Schluss nahe, dass es sich bei der Organisationsform Verein um ein Auslaufmodell handelt. Allerdings zeichnen sich einige Veränderungen in der Vereinslandschaft ab. So sind es tendenziell eher die bereits lange bestehenden freizeitbezogenen Vereine im ländlichen Raum, die von Mitgliederrückgängen berichten. Junge Vereine hingegen, etwa die des Handlungsfeldes Bürger- und Verbraucherinteressen, konnten in den vergangen Jahren häufig ihre Mitgliederzahlen erhöhen.

Die Vermutung liegt daher nahe, dass es vor allem kleine Vereine sind, die verstärkt neue Mitglieder gewinnen können. Dem ist jedoch nicht so. Bei fast jedem zweiten kleinen Verein (45,4 Prozent) hat sich die Zahl der Mitglieder seit 2012 nicht verändert, gestiegen ist sie bei etwa jedem dritten kleinen Verein (33,9 Prozent). Insgesamt scheint bei den großen Vereinen mehr Bewegung unter den Mitgliedern zu sein, denn sie haben sowohl die höchsten Anteile an Vereinen, die zusätzliche Mitglieder gewinnen konnten (41,4 Prozent), als auch die höchsten 
Tabelle 3.6 Entwicklung der Mitgliederzahlen seit 2012 nach Handlungsfeld (in Prozent)

\begin{tabular}{llll}
\hline & gestiegen & unverändert & gesunken \\
\hline Bürger-/Verbraucherinteressen & 51,3 & 35,6 & 13,1 \\
Wirtschafts-/Berufsverbände & 47,7 & 29,1 & 23,3 \\
Umwelt-/Naturschutz & 45,1 & 32,4 & 22,5 \\
Bevölkerungs-/Katastrophenschutz & 44,1 & 38,0 & 17,9 \\
Gesundheitswesen & 40,0 & 40,0 & 20,0 \\
Internationale Solidarität & 38,5 & 44,4 & 17,2 \\
Wissenschaft/Forschung & 37,7 & 41,3 & 21,0 \\
Bildung/Erziehung & 35,7 & 48,1 & 16,2 \\
Kirchen/religiöse Vereinigungen & 35,7 & 40,9 & 23,5 \\
\hline Gesamt & 35,2 & 42,5 & 22,3 \\
\hline Freizeit/Geselligkeit & 34,0 & 40,4 & 25,7 \\
Sport & 32,4 & 40,6 & 26,9 \\
Sonstiges & 32,4 & 49,0 & 18,7 \\
Gemeinschaftliche Versorgungsaufgaben & 32,4 & 52,9 & 14,7 \\
Kultur/Medien & 32,1 & 41,5 & 26,4 \\
Soziale Dienste & 31,2 & 48,0 & 20,7 \\
\hline
\end{tabular}

Quelle: ZiviZ-Survey 2017, nur Vereine, $\mathrm{n}=6461$ (gewichtet), davon fehlend: 600 .

Anteile an Vereinen, die Mitgliederverluste zu verzeichnen haben. Mitgliederrückgänge weisen vor allem Vereine in sehr kleinen Gemeinden auf. Die Abwanderung aus dem ländlichen Raum dürfte eine zentrale Ursache sein, da mit sinkenden Bevölkerungszahlen auch potenzielle Vereinsmitglieder und Engagierte verloren gehen. Betroffen sind daher im ländlichen Raum große und kleine Vereine gleichermaßen. Bei Vereinen in Dörfern bzw. in kleinen Gemeinden sind die Mitgliederzahlen am seltensten gestiegen (30,5 Prozent), dafür am häufigsten gesunken (25,5 Prozent). In mittleren Städten etwa ist es genau andersherum: Dort gibt es weniger Vereine mit gesunkenen Mitgliederzahlen (19,7 Prozent) und mehr Vereine, die neue Mitglieder gewonnen haben (39,2 Prozent). 
Tabelle 3.7 Entwicklung der Mitgliederzahlen seit 2012 nach Vereinsgröße (in Prozent)

\begin{tabular}{llll}
\hline & gestiegen & unverändert & gesunken \\
\hline klein (bis 100 Mitglieder) & 33,9 & 45,4 & 20,8 \\
mittelgroß (101 bis 300 Mitglieder) & 37,4 & 39,8 & 22,8 \\
groß (mehr als 300 Mitglieder) & 41,4 & 33,0 & 25,6 \\
\hline Gesamt & 35,9 & $\mathbf{4 2 , 1}$ & $\mathbf{2 2 , 0}$ \\
\hline
\end{tabular}

Quelle: ZiviZ-Survey 2017, nur Vereine, $\mathrm{n}=6461$ (gewichtet), davon fehlend: 895.

Tabelle 3.8 Entwicklung der Mitgliederzahlen seit 2012 nach Siedlungsgröße (in Prozent)

\begin{tabular}{llll}
\hline & gestiegen & unverändert & gesunken \\
\hline Dorf/Gemeinde & 30,5 & 43,9 & 25,6 \\
Kleinstadt & 34,8 & 43,0 & 22,2 \\
Mittelstadt & 39,2 & 41,1 & 19,7 \\
Großstadt & 35,7 & 42,4 & 21,9 \\
\hline Gesamt & $\mathbf{3 5 , 3}$ & $\mathbf{4 2 , 5}$ & $\mathbf{2 2 , 2}$ \\
\hline
\end{tabular}

Quelle: ZiviZ-Survey 2017, nur Vereine, $\mathrm{n}=6461$ (gewichtet), davon fehlend: 535 .

Freiwillig Engagierte: In fast allen Vereinen (94,o Prozent) ist mindestens eine Person freiwillig beziehungsweise ehrenamtlich engagiert. In allen Vereinen zusammen engagieren sich 24,2 Millionen Menschen ${ }^{6}$. Manche von ihnen übernehmen dauerhaft Ämter, zum Beispiel als Vorstandsmitglied, Trainer oder als Hausaufgabenpate. Manche engagieren sich nur gelegentlich, etwa beim von der freiwilligen Feuerwehr organisierten Sommerfest oder bei einem der Arbeitseinsätze der Sektionen des Deutschen Alpenvereins, bei denen unter anderem Berg- und Wanderwege in den Alpen neu angelegt oder in Stand gehalten werden. In zwei von drei Vereinen (64,5 Prozent) engagieren sich bis zu 20 Personen ehrenamtlich. Mehr als 50 Prozent der Freiwilligen engagieren sich in nur 13 Prozent der Vereine.

6 Damit ist die Zahl der in Vereinen engagierten Personen, die auf Basis der Daten des ZiviZSurveys ermittelt wurde, um Faktor 1,5 größer als die des Freiwilligensurveys. Diese Zahl ist ein realistischer Wert, denn eine Person kann gleichzeitig in mehreren Vereinen engagiert sein, insbesondere da es sich bei den im ZiviZ-Survey abgefragten Engagements nicht ausschließlich um Ehrenämter handelt, die einen dauerhaften und regelmäßigen Einsatz erfordern. 
Es ist nicht so, dass mitgliederstarke Vereine generell auch viele Engagierte binden. Es gibt nur einen geringen statistischen Zusammenhang zwischen der Zahl der Mitglieder und der der freiwillig Engagierten. Es sind auch nicht die Handlungsfelder mit den größten Vereinen, in denen die meisten Engagierten aktiv sind. Eine hohe Zahl an Engagierten können vor allem die Vereine des Bevölkerungsund Katastrophenschutzes binden. Hierzu zählen Feuerwehrvereine und andere Katastrophen- und Rettungsdienste wie zum Beispiel die Vereine der Deutschen Lebens-Rettungs-Gesellschaft (DLRG). In einem von drei Vereinen des Bevölkerungs- und Katastrophenschutzes (35,2 Prozent) sind mindestens 5o Personen engagiert. In vielen Fällen sind es deutlich mehr.

Die Zahl der Engagierten ist seit 2012 bei den meisten Vereinen (61,7 Prozent) stabil. Gestiegen sind die Engagiertenzahlen in 21,4 Prozent der Vereine, gesunken in 16,9 Prozent. Der Anteil der Vereine mit gestiegenen Engagiertenzahlen ist

Tabelle 3.9 Freiwillig Engagierte nach Handlungsfeld (in Prozent)

\begin{tabular}{llll}
\hline & $\begin{array}{l}\text { klein } \\
\text { (bis 20 En- } \\
\text { gagierte) }\end{array}$ & $\begin{array}{l}\text { mittelgroß } \\
(21-50 \mathrm{En}- \\
\text { gagierte) }\end{array}$ & $\begin{array}{l}\text { groß } \\
\text { (mehr als 50 En- } \\
\text { gagierte) }\end{array}$ \\
\hline Bevölkerungs-/Katastrophenschutz & 22,3 & 42,5 & 35,2 \\
Kirchen/religiöse Vereinigungen & 60,9 & 18,7 & 20,4 \\
Soziale Dienste & 60,5 & 22,0 & 17,5 \\
Wirtschafts-/Berufsverbände & 65,4 & 17,3 & 17,3 \\
Sport & 57,9 & 26,3 & 15,7 \\
Bürger-/Verbraucherinteressen & 60,4 & 25,5 & 14,1 \\
Freizeit/Geselligkeit & 66,7 & 20,0 & 13,2 \\
\hline Gesamt & 64,5 & 22,5 & 13,0 \\
\hline Gesundheitswesen & 73,0 & 16,9 & 10,1 \\
Kultur/Medien & 65,8 & 24,6 & 9,6 \\
Internationale Solidarität & 70,8 & 19,6 & 9,5 \\
Bildung/Erziehung & 76,0 & 15,2 & 8,8 \\
Umwelt-/Naturschutz & 63,3 & 28,2 & 8,5 \\
Sonstiges & 71,7 & 20,2 & 8,2 \\
Wissenschaft/Forschung & 70,8 & 23,3 & 5,8 \\
\hline
\end{tabular}

Quelle: ZiviZ-Survey 2017, nur Vereine mit freiwillig Engagierten, n = 6461 (gewichtet), davon fehlend: 790, ohne Gemeinschaftliche Versorgungsaufgaben, da zu wenig Fälle. 
also noch immer höher als der Anteil der Vereine, bei denen die Engagiertenzahlen gesunken sind. Da heute eine größere Zahl an Vereinen um Mitglieder und um Ehrenamtliche konkurriert, ist dies durchaus ein positiver Befund, insbesondere, da sich die Zahlen gegenüber dem ZiviZ-Survey 2012 nur geringfügig verändert haben (vgl. Abb. 3.2).

Eine besonders gute Entwicklung zeichnet sich bei den Vereinen der Sozialen Dienste und den Vereinen mit internationalen (Internationale Solidarität) oder religiösen (Kirchen/religiöse Vereinigungen) Bezügen ab. In diesen Handlungsfeldern hat etwa jeder dritte Verein heute mehr Engagierte als im Jahr 2012, obwohl schon der ZiviZ-Survey 2012 hier überdurchschnittliche Zuwächse an Engagierten dokumentierte. Auch haben diese Handlungsfelder vergleichsweise wenige Vereine mit rückläufigen Engagiertenzahlen. Neu ist die positive Entwicklung der Engagiertenzahlen in einem Drittel der Vereine der Bürger- und Verbraucherinteressen (31,9 Prozent). Im Jahr 2012 lag dieser Anteil noch bei 17,5 Prozent. Doch dort gibt es immerhin auch 14,6 Prozent, bei denen die Engagiertenzahlen rückläufig waren. Es ist naheliegend, dass die positive Entwicklung im Zusammenhang mit der starken Zuwanderung von Flüchtlingen in den Jahren 2015 und 2016 steht. Denn gerade im Handlungsfeld Bürger- und Verbraucherinteressen haben sich besonders viele Vereine in der Flüchtlingshilfe engagiert. Die größten Herausforderungen hinsichtlich der Bindung von freiwillig Engagierten bestehen offenbar für die beiden Handlungsfelder Sport sowie Freizeit und Geselligkeit. Es gibt dort

Abbildung 3.2 Entwicklung der Engagiertenzahlen (in Prozent)



Quelle: ZiviZ-Survey 2017, nur Vereine mit freiwillig Engagierten, $n=5959$ (gewichtet), davon fehlend: 624; ZiviZ-Survey 2012, n = 3422 (gewichtet), davon fehlend: 261. 
mehr Vereine mit rückläufigen Engagiertenzahlen als Vereine, die neue Engagierte gewinnen konnten. Positiv zu vermerken ist, dass sich seit 2012 wenig an diesem Verhältnis geändert hat. Anders sieht es bei den Kulturvereinen aus. Unter ihnen gibt es ebenfalls überdurchschnittlich viele mit rückläufigen und unterdurchschnittlich wenige mit gestiegenen Engagiertenzahlen. Erwähnenswert ist, dass es offenbar den kleinen Vereinen eher gelingt, Engagierte zu gewinnen und $\mathrm{zu}$ halten. Denn während 23,3 Prozent der kleinen Vereine mit weniger als 100 Mitgliedern heute mehr Engagierte haben als 2012 und auch nur 14,8 Prozent von ihnen berichten, dass die Engagiertenzahlen rückläufig sind, verhält es sich bei den großen Vereinen mit mehr als 300 Mitgliedern genau andersherum. 22,5 Prozent der großen Vereine berichten, dass die Engagiertenzahlen zurückgehen, und nur 18,6 Prozent, dass sie steigen. Die Stadt-Land-Problematik zeigt sich auch bei den Engagierten: Vereine in Dörfern und kleinen Gemeinden haben häufiger Rückgänge der Engagiertenzahlen (21,7 Prozent) und konnten seltener neue Engagierte

Tabelle 3.10 Entwicklung der Engagiertenzahlen seit 2012 nach Handlungsfeld (in Prozent)

\begin{tabular}{llll}
\hline & gestiegen & unverändert & gesunken \\
\hline Soziale Dienste & 34,3 & 56,3 & 9,5 \\
Bürger-/Verbraucherinteressen & 31,9 & 53,5 & 14,6 \\
Internationale Solidarität & 30,9 & 60,4 & 8,7 \\
Kirchen/religiöse Vereinigungen & 30,8 & 62,5 & 6,7 \\
Umwelt-/Naturschutz & 27,0 & 55,3 & 17,6 \\
Wirtschafts-/Berufsverbände & 26,9 & 59,7 & 13,4 \\
Bevölkerungs-/Katastrophenschutz & 26,4 & 49,7 & 23,9 \\
Bildung/Erziehung & 22,0 & 63,0 & 15,0 \\
Gesundheitswesen & 21,5 & 61,9 & 16,6 \\
\hline Gesamt & 21,3 & 61,6 & 17,1 \\
\hline Sonstiges & 20,1 & 68,3 & 11,6 \\
Kultur/Medien & 19,8 & 60,1 & 20,0 \\
Freizeit/Geselligkeit & 17,3 & 61,8 & 20,9 \\
Sport & 14,4 & 62,8 & 22,8 \\
Gemeinschaftliche Versorgungsaufgaben & 13,3 & 76,7 & 10,0 \\
\hline
\end{tabular}

Quelle: ZiviZ-Survey 2017, nur Vereine mit freiwillig Engagierten, $\mathrm{n}=5959$ (gewichtet), davon fehlend: 745, ohne Gemeinschaftliche Versorgungsaufgaben, da zu wenig Fälle. 
Tabelle 3.11 Entwicklung der Engagiertenzahlen seit 2012 nach Vereinsgröße (in Prozent)

\begin{tabular}{llll}
\hline & gestiegen & unverändert & gesunken \\
\hline klein (bis 100 Mitglieder) & 23,3 & 62,0 & 14,8 \\
mittelgroß (101 bis 300 Mitglieder) & 18,6 & 62,9 & 18,5 \\
groß (mehr als 300 Mitglieder) & 18,6 & 58,9 & 22,5 \\
\hline Gesamt & 21,4 & 61,7 & 17,0 \\
\hline
\end{tabular}

Quelle: ZiviZ-Survey 2017, nur Vereine mit freiwillig Engagierten (gewichtet), $\mathrm{n}=5$ 959, davon fehlend: 962.

Tabelle 3.12 Entwicklung der Engagiertenzahlen seit 2012 nach Siedlungsgröße (in Prozent)

\begin{tabular}{llll}
\hline & gestiegen & unverändert & gesunken \\
\hline Dorf/Gemeinde & 18,3 & 60,0 & 21,7 \\
Kleinstadt & 18,0 & 62,7 & 19,2 \\
Mittelstadt & 22,8 & 62,2 & 15,0 \\
Großstadt & 24,8 & 61,7 & 13,5 \\
\hline Gesamt & $\mathbf{2 1 , 5}$ & $\mathbf{6 1 , 7}$ & $\mathbf{1 6 , 8}$ \\
\hline
\end{tabular}

Quelle: ZiviZ-Survey 2017, nur Vereine mit freiwillig Engagierten (gewichtet), $n=5959$, davon fehlend: 689.

gewinnen (18,3 Prozent). Ganz anders sieht die Situation in den Großstädten aus. Bei 24,8 Prozent der Vereine in Großstädten sind seit 2012 neue Engagierte hinzugekommen, bei nur 13,5 Prozent sind sie gesunken.

Auch das Vereinsprofil hat einen Effekt auf die Entwicklungen der Engagiertenzahlen. Vereine, deren Rekrutierungsstrategie es zulässt, dass sich auch externe Personen engagieren, konnten nicht nur häufiger mehr Engagierte gewinnen, sondern haben auch seltener rückläufige Engagiertenzahlen als jene Vereine, deren Engagierte ausschließlich aus den eigenen Reihen stammen.

Trotz der stabilen bis positiven Entwicklung der Engagiertenzahlen gibt es eine ausgeprägte Problemwahrnehmung hinsichtlich der Herausforderungen, die im Zusammenhang mit der Mobilisierung und Bindung von Ehrenamtlichen bestehen. Insbesondere die Mobilisierung von Engagierten für dauerhafte Aktivitäten ist problematisch. Nur 13,5 Prozent der Vereine geben an, dass es einfach sei, für dauerhaftes Engagement Engagierte zu gewinnen. Zwei von drei Vereinen 
Tabelle 3.13 Entwicklung der Engagiertenzahlen seit 2012 nach Mitgliedschaft der Engagierten (in Prozent)

\begin{tabular}{llll}
\hline Unsere Engagierten sind... & gestiegen & unverändert & gesunken \\
\hline Mitglieder der Organisation & 16,7 & 65,0 & 18,3 \\
Mitglieder und Nichtmitglieder & 31,2 & 54,4 & 14,3 \\
keine Mitglieder der Organisation & 35,3 & 54,3 & 10,3 \\
\hline
\end{tabular}

Quelle: ZiviZ-Survey 2017, nur Vereine mit freiwillig Engagierten (gewichtet), $\mathrm{n}=5$ 959, davon fehlend: 693.

(61,6 Prozent) sehen das anders. Besonders schwierig ist es für Umweltschutzvereine. 70,1 Prozent von ihnen geben an, dass es schwierig ist, dauerhaft engagierte Personen zu finden. Ähnlich hoch sind die Anteile in den Handlungsfeldern Bürger- und Verbraucherinteressen (66,9 Prozent), Sport (64,7 Prozent), Internationale Solidarität (62,6 Prozent) und Bildung (62,6 Prozent).

Engagierte für kurzfristige Einsätze zu gewinnen, ist vergleichsweise wenig problematisch. Mehr als jeder zweite Verein (52,2 Prozent) findet für kurzfristige Engagements leicht Engagierte. Vor allem für Vereine der Handlungsfelder Kirchen und religiöse Vereinigungen, Bevölkerungs- und Katastrophenschutz sowie Internationale Solidarität ist es offenbar unproblematisch, für kurze Einsätze Engagierte zu mobilisieren.

Vor allem den großen Vereinen fällt es zunehmend schwer, dauerhaft Engagierte zu gewinnen und zu binden. Nur jeder zehnte Verein mit mehr als 300 Mitgliedern (10,9 Prozent) kann für dauerhafte Posten problemlos Engagierte mobilisieren. Für kleine Vereine ist es offenbar etwas leichter: 14,5 Prozent geben an, dass es leicht sei, dauerhaft Engagierte zu gewinnen. Zwar verneinen immerhin zwei von drei kleinen Vereinen (60,8 Prozent) diese Aussage, aber das sind immer noch etwas weniger als bei den großen Vereinen (65,1 Prozent). Hinsichtlich kurzfristiger Engagements spielt die Mitgliederzahl der Vereine eine geringere Rolle. Egal ob die Vereine groß, klein oder mittel sind: Es ist für jeden zweiten Verein leicht, Engagierte für kurze Einsätze zu mobilisieren. 
Tabelle 3.14 „Für dauerhafte Engagements ist es einfach, Engagierte zu gewinnen." (in Prozent)

\begin{tabular}{llll}
\hline & trifft (voll) zu & weder noch & trifft (gar) nicht zu \\
\hline Umwelt-/Naturschutz & 8,6 & 21,3 & 70,1 \\
Bürger-/Verbraucherinteressen & 9,3 & 23,8 & 66,9 \\
Bevölkerungs-/Katastrophenschutz & 10,1 & 29,8 & 60,1 \\
Sport & 10,4 & 25,0 & 64,7 \\
Gemeinschaftliche Versorgungsaufgaben & 11,4 & 28,6 & 60,0 \\
Internationale Solidarität & 12,3 & 25,2 & 62,6 \\
Bildung/Erziehung & 13,1 & 24,3 & 62,6 \\
\hline Gesamt & 13,5 & 24,9 & 61,6 \\
\hline Gesundheitswesen & 13,8 & 28,2 & 58,0 \\
Kultur/Medien & 14,0 & 25,4 & 60,6 \\
Kirchen/religiöse Vereinigungen & 14,0 & 29,0 & 57,0 \\
Freizeit/Geselligkeit & 17,2 & 23,3 & 59,5 \\
Sonstiges & 17,4 & 21,9 & 60,7 \\
Soziale Dienste & 19,3 & 23,0 & 57,7 \\
Wirtschafts-/Berufsverbände & 21,1 & 29,6 & 49,3 \\
\hline
\end{tabular}

Quelle: ZiviZ-Survey 2017, nur Vereine mit freiwillig Engagierten (gewichtet), $\mathrm{n}=5$ 959, davon fehlend: 466.

Tabelle 3.15 „Für dauerhafte Engagements ist es einfach, Engagierte zu gewinnen.”

\begin{tabular}{llll}
\hline & trifft (voll) zu & weder noch & trifft (gar) nicht zu \\
\hline klein (bis 100 Mitglieder) & 14,5 & 24,7 & 60,8 \\
mittelgroß (101 bis 300 Mitglieder) & 12,9 & 25,9 & 61,2 \\
groß (mehr als 300 Mitglieder) & 10,9 & 24,0 & 65,1 \\
\hline Gesamt & 13,5 & 24,9 & 61,6 \\
\hline
\end{tabular}

Quelle: ZiviZ-Survey 2017, nur Vereine mit freiwillig Engagierten (gewichtet), $\mathrm{n}=5$ 959, davon fehlend: 710. 
Tabelle 3.16 „Für kurzfristige Engagements ist es einfach, Engagierte zu gewinnen.”

\begin{tabular}{llll}
\hline & trifft (voll) zu & weder noch & trifft (gar) nicht zu \\
\hline klein (bis 100 Mitglieder) & 52,1 & 27,3 & 20,6 \\
mittelgroß (101 bis 300 Mitglieder) & 52,5 & 30,2 & 17,3 \\
groß (mehr als 300 Mitglieder) & 51,9 & 27,6 & 20,4 \\
\hline Gesamt & 52,2 & 28,1 & 19,7 \\
\hline
\end{tabular}

Quelle: ZiviZ-Survey 2017, nur Vereine mit freiwillig Engagierten (gewichtet), $\mathrm{n}=5$ 959, davon fehlend: 684.

Integrationsbeitrag: Vereine bieten ein breites Spektrum an Angeboten und Maßnahmen für Zielgruppen mit besonderen Integrationsbedarfen. Allgemein an sozial benachteiligte Menschen richten sich 25,8 Prozent der Vereine mit ihren Aktivitäten. Konkrete Zielgruppen sind etwa Menschen mit Behinderungen, MigrantInnen oder Flüchtlinge. Vereine bieten ihnen unter anderem sportliche oder kulturelle Betätigungsmöglichkeiten, aber auch konkrete Förder- und Weiterbildungsmaßnahmen. Sie koordinieren beispielsweise Lese- und Hausaufgabenpaten oder bieten Hilfen zum (Wieder-)Einstieg in den Arbeitsmarkt, etwa durch gezielte Qualifizierungsmaßnahmen und Bewerbertrainings. Jeder fünfte Verein macht Angebote für Menschen mit Behinderungen (19,4 Prozent), MigrantInnen (19,1 Prozent) oder Flüchtlinge (18 Prozent). Von Vereinen wurde und wird viel Engagement in der Flüchtlingshilfe geleistet. So engagieren sich 14,o Prozent der Vereine in der Flüchtlingshilfe. Die meisten haben diese Aktivitäten allerdings erst aufgenommen, als der Bedarf am größten war, nämlich in den Jahren 2015 und 2016. Neben Hilfsangeboten wie Sachspenden und Unterstützung der Flüchtlinge bei Alltagsangelegenheiten organisieren sie auch Angebote zum interkulturellen Austausch vor Ort, durch die sie Einheimischen und Flüchtlingen bei gemeinsamen Unternehmungen wie Kochen, Fußballspielen oder Gärtnern Gelegenheiten bieten, sich kennenzulernen und auszutauschen.

Vor dem Hintergrund der aktuellen Integrationsdebatte ist es von besonderem Interesse, in welchem Maße Vereine bestimme Zielgruppen nicht nur als passive Angebots- und Hilfeempfänger ansprechen, sondern auch Teilhabe in Form von Mitgliedschaften und Engagements ermöglichen. Bislang sind beispielsweise Menschen mit Migrationshintergrund wenig in die Vereine der Mehrheitsgesellschaft eingebunden. Meist teilen Mitglieder und freiwillig Engagierte Sprache, Religion und Nationalität. In drei von vier Vereinen ( 72,1 Prozent) haben die Mitglieder eine ähnliche kulturelle Herkunft. Ebenso sieht es unter den freiwillig Engagierten aus, die in 79,2 Prozent der Vereine eine ähnliche kulturelle Herkunft 
Tabelle 3.17 Vereine, die in der Flüchtlingshilfe aktiv sind oder waren, nach Handlungsfeld (in Prozent)

\begin{tabular}{ll}
\hline Soziale Dienste & 27,3 \\
Bürger-/Verbraucherinteressen & 25,5 \\
Kirchen/religiöse Vereinigungen & 24,9 \\
Bildung/Erziehung & 19,5 \\
Bevölkerungs-/Katastrophenschutz & 18,9 \\
Internationale Solidarität & 18,6 \\
Sonstiges & 17,2 \\
\hline Gesamt & 13,9 \\
\hline Gemeinschaftliche Versorgungsaufgaben & 11,8 \\
Sport & 10,8 \\
Gesundheitswesen & 10,7 \\
Wirtschafts-/Berufsverbände & 10,1 \\
Kultur/Medien & 8,2 \\
Wissenschaft/Forschung & 7,3 \\
Freizeit/Geselligkeit & 3,1 \\
Umwelt-/Naturschutz & 1,6 \\
\hline
\end{tabular}

Quelle: ZiviZ-Survey 2017, nur Vereine, N = 6461 (gewichtet), davon fehlend: 386 .

haben. Jüngere Vereine, insbesondere jene im städtischen Raum, sind hinsichtlich der interkulturellen Öffnung etwas besser aufgestellt als ein Großteil der älteren Vereine, insbesondere jene im ländlichen Raum, wodurch sich auch Unterschiede zwischen den Handlungsfeldern erklären. So sind es vor allem Vereine des Bevölkerungs- und Katastrophenschutzes, aber auch Kulturvereine, in denen Mitglieder und Engagierte überdurchschnittlich homogen aufgestellt sind. Dort, wo nur wenige MigrantInnen leben, sind sie entsprechend seltener in Vereine eingebunden, wie etwa in den meisten ländlichen Räumen, was sich selbstredend auch in der kulturellen Vielfalt der Vereine widerspiegelt. 82,1 Prozent der Vereine in kleinen Kommunen berichten von einer homogenen Mitgliederstruktur. Bei den Vereinen in den Großstädten liegt dieser Anteil bei 64,8 Prozent. Ähnlich verhält es sich bei den Engagierten: 86,2 Prozent der Vereine im ländlichen Raum berichten, dass ihre Engagierten eine ähnliche kulturelle Herkunft haben. In den Großstädten sind es nur 74,o Prozent. 
Abbildung 3.3 „Unsere Engagierten/Mitglieder haben eine ähnliche kulturelle Herkunft." (in Prozent)

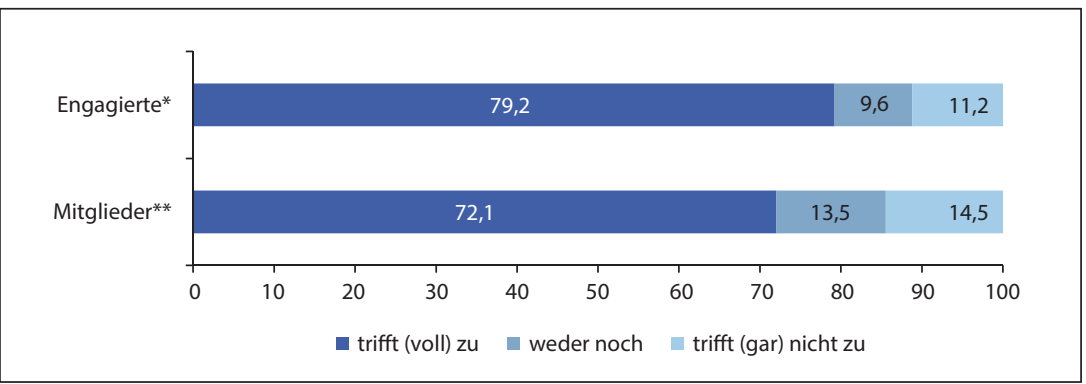

Quelle: ZiviZ-Survey 2017, ${ }^{*}$ nur Vereine, $\mathrm{n}=6461$ (gewichtet), davon fehlend: 489; ${ }^{* *}$ nur Vereine mit freiwillig Engagierten, $n=5959$ (gewichtet), davon fehlend: 331 .

Ähnlich verhält es sich mit der zunehmenden Öffnung der Vereine für MigrantInnen. In Dörfern und kleinen Gemeinden berichten nur 1,1 Prozent der Vereine, dass sie zunehmend MigrantInnen unter den Engagierten haben. In den Großstädten sind es hingegen 5,o Prozent. Obgleich die Bevölkerungsverteilung ein auschlaggebender Grund ist, mögen hier auch andere Faktoren hineinspielen, etwa das gezielte Anwerben von MigrantInnen für die Vereinsarbeit als Mitglieder. Nicht einmal annähernd jeder zehnte Verein (6,4 Prozent) versucht gezielt, Mitglieder mit Migrationshintergrund zu gewinnen, wodurch der bislang nur geringe Zuwachs von MigrantInnen unter den Engagierten in den meisten Vereinen begründet sein dürfte. Da Engagierte oftmals aus den Reihen der eigenen Mitglieder rekrutiert werden, haben jene Vereine, die bereits bei den Mitgliedern verstärkt auf MigrantInnen setzen, auch nachfolgend eher Engagierte mit Migrationshintergrund. Insgesamt geben nur 3,3 Prozent der Vereine an, dass sich bei ihnen zunehmend Menschen mit Migrationshintergrund engagieren. Bei den Vereinen, die sich um Mitglieder mit Migrationshintergrund bemühen, ist der Anteil mit 13,1 Prozent jedoch vier Mal so hoch. In den Großstädten werben 7,2 Prozent der Vereine um MigrantInnen als Mitglieder, in kleinen Gemeinden nur 4,2 Prozent. Die Vereinsgröße ist für die Gewinnung migrantischer Engagierter nur indirekt von Bedeutung. Egal wie groß die Vereine sind, der Wert ändert sich kaum. Ein indirekter Einfluss ergibt sich dadurch, dass jene Vereine, die AnsprechpartnerInnen zur Koordinierung freiwillig Engagierter haben, auch häufiger erfolgreicher bei der Mobilisierung von Engagierten mit Migrationshintergrund sind. Solche AnsprechpartnerInnen wiederum haben häufiger große Vereine. 
Abbildung 3.4 "Bei uns engagieren sich zunehmend Menschen mit Migrationshintergrund" (in Prozent)

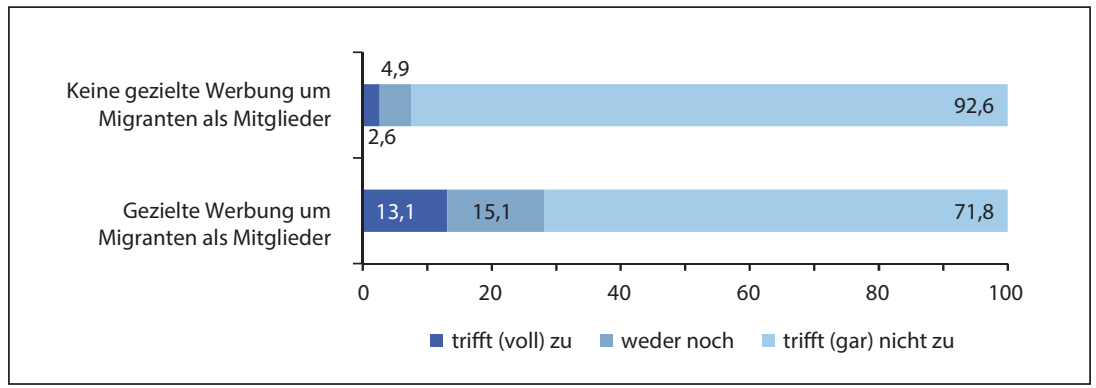

Quelle: ZiviZ-Survey 2017, nur Vereine mit freiwillig Engagierten, n = 5959 (gewichtet), davon fehlend: 708.

Vereine als Bildungsakteure: Die Bedeutung von Vereinen für das Bildungswesen zeigt sich nicht nur darin, dass Bildung heute nach Sport die meisten Vereine zählt, sondern auch in den zahlreichen Bildungs- und Qualifizierungsangeboten von Vereinen für ihre Mitglieder, Engagierten oder für externe NutzerInnen. 39,2 Prozent der Vereine stellen eigene Bildungsangebote bereit und ergänzen damit das schulische und außerschulische Bildungsangebot und befördern das lebenslange Lernen, vor allem in außerschulischen Kontexten. In der Realität dürften es sogar noch mehr sein. Offenbar nehmen sich nicht alle Vereine, die Bildungsangebote machen, als Bildungsakteure wahr. Beispielsweise gibt nicht einmal jeder dritte Verein des Bevölkerungs- und Katastrophenschutzes (28,1 Prozent) an, dass er Bildungsangebote bereitstellt, obwohl ein Feuerwehrverein oder ein Verein der Deutschen Lebens-Rettungs-Gesellschaft die Engagierten in der Regel zunächst qualifiziert, bevor er sie einsetzt. Auch im Sport ist der Anteil mit 27,4 Prozent überraschend klein, denn ein Großteil der Sportvereine und -verbände bietet nicht nur Sport- und Bewegungsangebote (die bereits ein Bildungsangebot für sich darstellen), sondern auch umfangreiche Ausbildungsangebote für TrainerInnen und ÜbungsleiterInnen an.

Das Leistungsspektrum der Bildungsangebote ist weit und reicht von Hausaufgabenhilfen und Nachhilfeangeboten über Computertrainings für SeniorInnen, Sicherheitstrainings für RadfahrerInnen und andere VerkehrsteilnehmerInnen, politische Bildungsangebote (zum Beispiel Informationsveranstaltungen und Podiumsdiskussionen) bis hin zu Qualifizierungsangeboten und Bewerbertrainings für Arbeitssuchende sowie Schulungen und Fortbildungen für freiwillig Engagierte (zum Beispiel Erste-Hilfe-Kurse, Trainerausbildung). Am weitesten verbreitet 
Tabelle 3.18 Häufigkeit der Bereitstellung folgender ausgewählter Bildungsangebote (Mehrfachnennungen möglich, in Prozent)

\begin{tabular}{llll}
\hline & oft & manchmal & nie \\
\hline Sport-/Bewegungsangebote & $\mathbf{2 8 , 6}$ & 20,0 & 51,4 \\
Kulturelle Bildungsangebote & $\mathbf{2 4 , 6}$ & 30,8 & 44,6 \\
Allgemeine Weiterbildungs-/Qualifizierungsangebote & $\mathbf{2 4 , 1}$ & 37,8 & $\mathbf{3 8 , 1}$ \\
Hortangebote/Nachmittagsbetreuung & 16,1 & 8,9 & $\mathbf{7 5 , 0}$ \\
Berufliche Weiterbildungs-/Qualifizierungsangebote & 14,0 & 19,0 & $\mathbf{6 7 , 0}$ \\
Sprachunterricht/Sprachförderung & 13,8 & 13,5 & $\mathbf{7 2 , 6}$ \\
Museums-/erlebnispädagogische Angebote & 10,7 & 30,3 & $\mathbf{5 9 , 0}$ \\
Hilfe bei der Integration in den Arbeitsmarkt & 9,5 & 18,0 & $\mathbf{7 2 , 5}$ \\
Bildungsreisen & 6,8 & $\mathbf{2 7 , 2}$ & $\mathbf{6 6 , 0}$ \\
\hline
\end{tabular}

Quelle: ZiviZ-Survey 2017 (nur Vereine, die Betreuungsangebote bereitstellen: $n=2416$; Mehrfachnennungen).

sind Sport- und Bewegungs- sowie kulturelle Bildungsangebote, aber auch diverse allgemeine Weiterbildungs- und Qualifizierungsangebote. Da der Zugang zu Bildung und Qualifikationen die gesellschaftliche Integration befördern, leisten Vereine auch hiermit wichtige Integrationsbeiträge.

\subsection{Sportvereine als Prototyp für das Vereinswesen ${ }^{7}$}

Die folgenden Ausführungen und Daten über die Sportvereinsentwicklung beziehen sich ausschließlich auf die 90000 verbandlich organisierten und eingetragenen Sportvereine (e.V.) in Deutschland. Diese sind mit über 27 Millionen Mitgliedschaften über einen regionalen (Landessportbünde) und sportartspezifischen (Spitzensportverbände) Bezug in den Deutschen Olympischen Sportbund (DOSB) als Dachverband des Sports rechtlich eingebunden.

Zentrale Grundlage für die Analyse bilden die Daten der Sportentwicklungsberichte (SEB), die regelmäßig als umfangreiche Vereinsbefragung von der Deutschen Sporthochschule Köln, Institut für Sportökonomie und Sportmanagement, unter der Leitung von Univ.-Prof. Dr. Christoph Breuer erhoben werden. Damit

7 Dieses Unterkapitel wurde verfasst von Boris Rump, DOSB. 
ergänzen und spezifizieren die Daten aus dem SEB die vorangestellten Ergebnisse aus dem ZiviZ-Survey über den Bereich Sport, in deren Analyse vor allen Dingen auch jene (Sport-)Vereine und weitere Organisationen (Stiftungen, Träger der Kinder- und Jugendhilfe, gGmbHs) abgebildet sind, die außerhalb der verbandlichen Sportvereinsstruktur liegen, aber ihr Haupttätigkeitsfeld im Sport sehen.

„Der vermeintliche „Niedergang“ des Sportvereins wird seit knapp 6o Jahren vorausgesagt (vgl. Croon 1959). Die Hauptargumente sind (1) Steuerungs- und Effizienzprobleme ehrenamtlicher Arbeit, die in einer komplexeren und sich dynamisch verändernden Vereinsumwelt zu einem größeren Organisationsproblem werden, sowie (2) eine vermeintlich nachlassende Bereitschaft zum ehrenamtlichen Engagement, die Umweltanpassungen notwendig erscheinen lässt (zum Überblick der Argumentation vgl. Breuer 2005). Paradoxerweise gingen die Behauptungen zum „Niedergang“ mit einer Erfolgsgeschichte des Sportvereins einher. Allen Argumenten zum Trotz ist sowohl die Anzahl der Sportvereine als auch die Anzahl der Mitgliedschaften in Sportvereinen seit 2000 gestiegen und verharren seither relativ stabil auf hohem Niveau." (Breuer und Feiler, 2017b).

„Daneben werden in Sportvereinen systematische Gemeinwohlbeiträge erbracht, wie etwa im Bereich Gesundheitsförderung, Prävention und Rehabilitation, Offener Ganztag oder Integrationsarbeit. Vermutlich konnten sich zahlreiche Behauptungen und

Tabelle 3.19 Entwicklung des Deutschen Sports 2000-2016

\begin{tabular}{lllllll}
\hline Jahr & Sportvereine & Mitgliedschaften* & & Jahr & Sportvereine & Mitgliedschaften* \\
\cline { 1 - 3 } 2000 & 87717 & 26812757 & & 2009 & 90897 & 27553516 \\
2001 & 88531 & 26838739 & & 2010 & 91148 & 27636026 \\
2002 & 88960 & 26897744 & & 2011 & 91250 & 27675461 \\
2003 & 89307 & 26909924 & 2012 & 91080 & 27774550 \\
2004 & 90305 & 27544736 & 2013 & 90784 & 27992386 \\
2005 & 89870 & 27220905 & 2014 & 90802 & 27775763 \\
2006 & 88637 & 27315184 & 2015 & 90240 & 27272854 \\
2007 & 91091 & 27341194 & 2016 & 90025 & 27520141 \\
2008 & 90775 & 27440906 & 2017 & 89594 & 27402981 \\
\hline
\end{tabular}

*Innerhalb der Bestandserhebung ist zu berücksichtigen, dass es Personen gibt, die in mehr als einem Sportverein Mitglied sind und daher in der Statistik mehrfach erfasst werden.

Quelle: DOSB-Bestandserhebung 2017. 
Spekulationen um die Zukunft des Sportvereins nur so lange halten, da sich mangels Datengrundlage entsprechende Thesen weder bekräftigen noch widerlegen ließen." (vgl. Breuer und Feiler, 2017b).

Befunde aus dem Sportentwicklungsbericht 2015/2016: ${ }^{8}$ Die Sportvereine in Deutschland erweisen sich nach wie vor als anpassungsfähige Stabilitätselemente in einer sich schnell wandelnden Gesellschaft. Neueste Befunde des Sportentwicklungsberichts zeigen, dass Sportvereine mit dem demografischen Wandel, aber auch mit der offenen Ganztagesschule und gymnasialen Schulzeitverkürzung (G8) immer besser zurechtkommen. Auch haben sie eine klarere Gesamtperspektive, wohin ihre Entwicklung gehen soll. 29 Prozent engagieren sich in besonderer Weise für Flüchtlinge. 35 Prozent der Vereine ermöglichen Menschen mit Behinderungen Sport. 52 Prozent der Vereine engagieren sich mittlerweile in besonderer Weise für die Prävention sexualisierter Gewalt im Verein. Die gemeinwohlorientierte Grundausrichtung der Sportvereine bleibt stabil: Sie bekennen sich weiterhin in hohem Maße zu Wertevermittlung, einem preisgünstigen Sportangebot sowie gleichberechtigter Partizipation. Zudem legen sie Wert auf die Qualifizierung ihrer TrainerInnen. 8 Prozent der Vereine bzw. 7200 Vereine insgesamt sind überdies als Träger der freien Jugendhilfe anerkannt.

Der Anteil der Vereine, die sich in besonderer Weise im Gesundheitsbereich engagieren, pendelt sich bei etwas über 26 Prozent ein. Auffallend ist, dass, entgegen dem leicht rückläufigen Trend bei den Gesundheitssportangeboten, der Anteil der Rehabilitationsangebote an allen Sportangeboten wächst.

Die finanzielle Situation der Sportvereine in Deutschland ist stabil. Die Wahrnehmung der Finanzsituation als Problem des Vereins ist im Durchschnitt sogar signifikant gesunken. Die Einführung des gesetzlichen Mindestlohns bereitet 6 Prozent der Vereine größere Probleme.

Im Hinblick auf Verfügbarkeit, Zustand oder Eignung von Sportanlagen zeigt sich keine Problemverschärfung, jedoch auch keine Verbesserung der Situation. Blickt man auf die Mitglieder (indem man den Datensatz so gewichtet, dass er für die Sportvereinsmitglieder in Deutschland anstatt für die Sportvereine repräsentativ ist), so treten Probleme der Verfügbarkeit sowie der Zustände von Sportanlagen hervor. Dies ergibt sich daraus, dass größere Sportvereine stärker von Sportstättenproblemen betroffen sind. Bei der Diskussion über die Sportstättenprobleme ist zu berücksichtigen, dass Sporthallen erst im Laufe der Erhebung sowie danach verstärkt als Flüchtlingsunterkünfte genutzt wurden und diese besondere Problematik daher nur teilweise erfasst werden konnte. Zum Zeitpunkt der

8 Der folgende Textabschnitt ist nahezu wortwörtlich aus dem Sportentwicklungsbericht 2015/2016 übernommen (siehe Breuer und Feiler 2017a, S. 15f.) 
Erhebung (September bis Dezember 2015) gaben gut 3400 der 90 ooo Vereine an, von ihnen genutzte Sportanlagen seien in den vergangenen zwei Jahren als Flüchtlingsunterkünfte genutzt worden.

Hauptproblem der Sportvereine bleibt die Bereitschaft zum ehrenamtlichen Engagement. Zwar liegen keine signifikanten Veränderungen der Anzahl an Engagierten in formalen Positionen auf der Vorstands- oder Ausführungsebene (zum Beispiel SchiedsrichterInnen, ÜbungsleiterInnen) vor, doch hat sich das wahrgenommene Problem, ehrenamtliche Funktionsträger zu gewinnen bzw. zu binden, weiter verschärft. Zudem ist die Anzahl freiwilliger HelferInnen in den Vereinen leicht rückläufig (minus 4,4 Prozent gegenüber dem Jahr 2013).

Trotz der bedeutenden Gemeinwohlleistungen der Sportvereine sollte folgenden Befunden Aufmerksamkeit geschenkt werden: 13 Prozent der Vereine stimmen der Aussage gar nicht zu, dass sie sich für die Prävention sexualisierter Gewalt engagieren. Weiterhin stimmen 7 Prozent der Vereine der Aussage gar nicht $\mathrm{zu}$, dass sie sich stark in der Jugendarbeit engagieren. 4 Prozent der Vereine stimmen der Aussage gar nicht zu, dass sie Familien Sport ermöglichen und familienfreundlich sein wollen, und 3 Prozent der Vereine stimmen gar nicht $\mathrm{zu}$, sich für die gleichberechtigte Partizipation von Mädchen/Frauen und Jungen/Männern zu engagieren. Lediglich 2 Prozent der Vereine stimmen der Aussage gar nicht $\mathrm{zu}$, dass sie Menschen mit Migrationshintergrund Sport ermöglichen wollen. Insgesamt ist zu prüfen, ob diese Befunde auf eine Konzentration der entsprechenden Vereine auf ihre Kernaufgaben schließen lassen, ob die Strategie- und Kommunikationsarbeit der Verbände diese Vereine nicht erreicht oder ob andere Gründe für die Ablehnung vorliegen (Breuer und Feiler 2017a, S. 15f.).

Neben der insgesamt beschriebenen stabilen Entwicklung der Sportvereinslandschaft und der weiter wachsenden Angebotsvielfalt zeigen sowohl die aktuellen Befunde als auch die Querschnittsanalyse von 2005 bis 2015 aus dem Sportentwicklungsbericht 2015/2016 deutlich auf, in welchen Bereichen die Herausforderungen und Probleme innerhalb der Sportvereinsentwicklung liegen. So hat sich in den vergangenen zwölf Jahren der Anteil an Sportvereinen in Deutschland, die mit mindestens einem existentiellen Problem zu kämpfen haben, mehr als verdoppelt - von 15 Prozent im Jahr 2005 auf 35 Prozent im Jahr 2015 (Breuer und Feiler 2017b. S. 247).

Wie oben bereits angesprochen, sind Probleme im Bereich der Gewinnung und Bindung von ehrenamtlichen Funktionsträgern von allen möglichen existenzbedrohenden Problemen in den letzten Jahren absolut am stärksten gewachsen - gleiches gilt nahezu auch für den Aspekt der Gewinnung und Bindung von TrainerInnen sowie ÜbungsleiterInnen. Damit sind automatisch neue Strategien zur Personalentwicklung in Sportvereinen in den Mittelpunkt der Arbeit in den Sportverbänden und Vereinen selbst gerückt. Wichtigstes Ziel ist es, die kon- 
tinuierliche Förderung und Entwicklung von langfristigem Engagement in den Organisationen sicherzustellen und Maßnahmen zur Bindung und Gewinnung von ehrenamtlichen und freiwilligen MitarbeiterInnen innerhalb der Vereinsarbeit stärker als bisher zu implementieren.

\subsection{Stiftungen ${ }^{9}$}

Stiftungen sind eine wichtige Säule der Zivilgesellschaft. Vielfach sehen sie sich als Thementreiber und Innovatoren. Doch ebenso wenig wie es den Verein gibt, kann von der Stiftung gesprochen werden. Der Begriff Stiftung ist im Gesetz nicht definiert. Auch wenn somit nicht automatisch eine bestimmte Rechtsform mit dem Begriff der Stiftung einhergeht, verfügen Stiftungen über einheitliche charakteristische Merkmale. Die Stiftung ist gekennzeichnet als Vermögensmasse, die einem bestimmten Zweck, meist einem gemeinnützigen, auf Dauer gewidmet ist. Welche Zwecke die Stiftung verfolgt und wie ihre innere Organisation aussieht, legen die Stiftenden in der Satzung fest. Es gibt rechtsfähige und nicht rechtsfähige Stiftungen wie zum Beispiel die Treuhandstiftungen. Klassisches Instrument zur Verwirklichung eines auf Dauer angelegten Zwecks ist die rechtsfähige Stiftung des bürgerlichen Rechts. Ihre Entstehungsvoraussetzungen sind in den $\$ \$ 80 \mathrm{ff}$. des Bürgerlichen Gesetzbuches (BGB) geregelt, die durch die Landesstiftungsgesetze ausgefüllt werden. Die rechtsfähige Stiftung des bürgerlichen Rechts unterscheidet sich von sonstigen juristischen Personen des Privatrechts durch ihre fehlende Verbandsstruktur: Sie hat keine Mitglieder oder EigentümerInnen.

Weitere Rechtsformen sind der Stiftungsverein oder die Stiftungs-GmbH. Ferner wird je nach zugrunde liegendem Rechtsbereich zwischen privaten und öffentlich-rechtlichen Stiftungen sowie kirchlichen Stiftungen unterschieden, wobei letztere sowohl privat- als auch öffentlich-rechtlich organisiert sein können. Weitere Unterscheidungen orientieren sich am Stiftenden, dem Zweck (zum Beispiel Familienstiftung) oder der Art der Zweckerfüllung (zum Beispiel Trägerstiftung).

Die wichtigsten Kennzahlen zum Stiftungssektor: Der Stiftungssektor ist in den vergangenen 15 Jahren stark gewachsen: Zwischen 2001 und 2016 wurden jährlich im Schnitt knapp 800 rechtsfähige Stiftungen des bürgerlichen Rechts gegründet (Bundesverband Deutscher Stiftungen 2017, S. 23). Im Jahr 2017 wurden insgesamt 549 Stiftungen dieser Rechtsform neu errichtet (Abb. 3.5). Damit liegt ihre Zahl bei nunmehr 22274 (Stichtag 31. Dezember 2017). 95 Prozent aller rechtsfähigen

9 Dieses Unterkapitel wurde verfasst von Antje Bischoff und Ralf Krebstakies, Bundesverband Deutscher Stiftungen. 


\section{Stiftungstypologie}

Bürgerstiftung: Bürgerstiftungen sind gemeinnützige Stiftungen von BürgerInnen für BürgerInnen, deren Stiftungszweck möglichst breit gefasst ist und dessen Verwirklichung in einem geografisch begrenzten Raum erfolgt.

Familienstiftung: Familienstiftungen dienen ihrem Zweck nach überwiegend dem Interesse der Mitglieder einer oder mehrerer Familien. Die für eine Steuerbegünstigung erforderliche Förderung der Allgemeinheit liegt bei einer reinen Familienstiftung nicht vor. Sie wird daher auch als privatnützige Stiftung bezeichnet.

Gemeinnützige Stiftung: Eine Stiftung ist gemeinnützig, wenn ihr Zweck darauf gerichtet ist, die Allgemeinheit auf materiellem, geistigem oder sittlichem Gebiet selbstlos zu fördern. Die Anerkennung als gemeinnützig erfolgt durch die Finanzbehörden. Mit dem Status der Gemeinnützigkeit ist regelmäßig die Steuerbefreiung der Stiftung verbunden. Auch sind gemeinnützige Stiftungen berechtigt, Spenden entgegenzunehmen.

Kirchliche Stiftung: Eine kirchliche Stiftung ist eine Stiftung, deren Zweck überwiegend kirchlichen Aufgaben dient. Die Bestimmung als kirchliche Stiftung hängt vom Stifterwillen und von der Zustimmung der Kirche ab.

Operative Stiftung $\leftrightarrow$ Förderstiftung: Eine operative Stiftung führt eigene Projekte durch, bezweckt also nicht die Förderung fremder Projekte bzw. von Personen oder anderen gemeinwohlorientierten Organisationen (Förderstiftung).

Trägerstiftung (oder sog. Anstaltsträgerstiftung): Trägerstiftungen verwirklichen ihren Zweck in der Regel vornehmlich durch von ihnen betriebene Einrichtungen wie Krankenhäuser, Pflegeeinrichtungen, Museen oder Forschungszentren, deren Art den Zweck der Stiftung vorgibt. Neben den Erträgen aus dem Anlagevermögen finanzieren sich Trägerstiftungen über öffentliche Zuwendungen, aus ihren Dienstleistungen sowie Pflegesätzen. Treuhandstiftung $\leftrightarrow$ rechtsfähige Stiftung: Eine Treuhandstiftung, auch als unselbstständige, nichtrechtsfähige oder fiduziarische Stiftung bezeichnet, wird durch einen Vertrag zwischen dem Stifter und dem Treuhänder (Träger) errichtet. Der Stifter überträgt das Stiftungsvermögen dem Treuhänder, der es getrennt von einem eigenen Vermögen gemäß den Satzungsbestimmungen der Stiftung verwaltet. Anders als eine rechtsfähige Stiftung verfügt eine Treuhandstiftung nicht über eine eigene Rechtspersönlichkeit.

Öffentlich-rechtliche Stiftung: Stiftungen des öffentlichen Rechts werden von staatlicher Seite durch einen Stiftungsakt, insbesondere per Gesetz, errichtet und verfolgen Zwecke, die von einem besonderen öffentlichen Interesse sind.

Öffentliche Stiftung des bürgerlichen Rechts: Eine öffentliche Stiftung des bürgerlichen Rechts wird nach den Regeln des Privatrechts errichtet. Der Zusatz „öffentlich“ wird vor allem in Bayern und Baden-Württemberg verwendet und kennzeichnet Stiftungen, die Zwecke verfolgen, die zumindest teilweise dem Gemeinwohl dienen. Eine öffentliche Stiftung des bürgerlichen Rechts ist meistens, aber nicht notwendigerweise, gemeinnützig.

Unternehmensstiftung: Eine Unternehmensstiftung ist eine Stiftung, für die ein Unternehmen das Stiftungsvermögen und gegebenenfalls laufende Mittel bereitstellt.

Unternehmensverbundene Stiftung: Unternehmensverbundene Stiftungen halten Anteile an Unternehmen oder betreiben selbst ein Unternehmen. Sie werden häufig als Instrument zur Regelung der Unternehmensnachfolge eingesetzt.

Verbrauchsstiftung: Eine Verbrauchsstiftung nennt man eine Stiftung, deren Grundstockvermögen nach dem Willen des Stifters in einer bestimmten Zeitspanne ganz oder zum Teil für die Verwirklichung des Stiftungszwecks eingesetzt werden soll. 
Abbildung 3.5 Stiftungserrichtungen 1990-2017

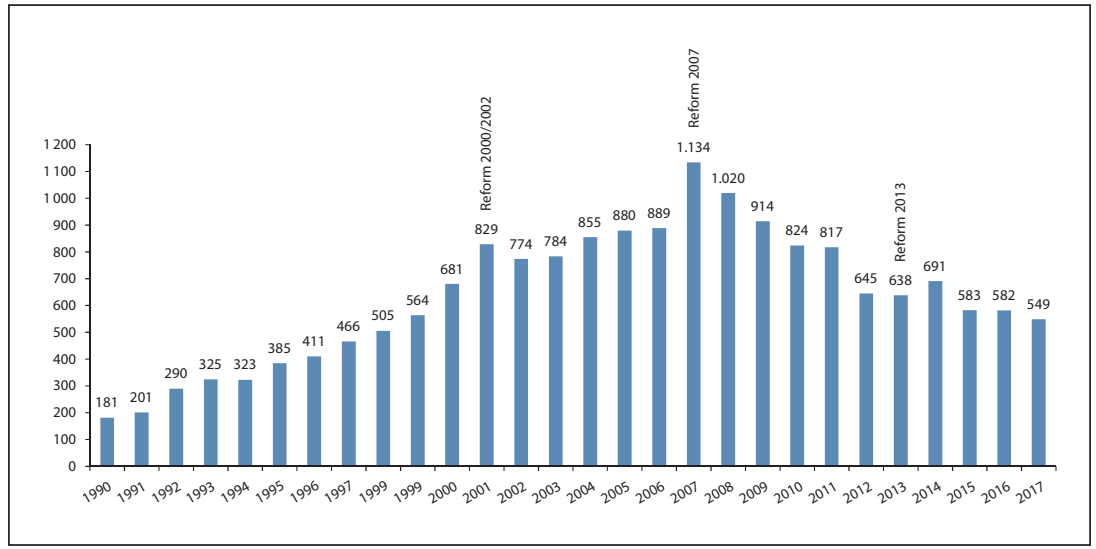

Quelle: Jährliche Umfrage unter den Stiftungsaufsichtsbehörden, Stichtag jeweils 31. Dezember.

Stiftungen bürgerlichen Rechts verfolgen steuerbegünstigte Zwecke (Bundesverband Deutscher Stiftungen 2017, S. 25).

$\mathrm{Zu}$ den weiteren Rechtsformen gibt es keine genauen Zahlen. In der Datenbank des Bundesverbandes Deutscher Stiftungen sind derzeit 5534 Treuhandstiftungen erfasst. Ferner weist die Datenbank 2106 Trägerstiftungen, 1083 rechtsfähige kirchliche Stiftungen und 798 rechtsfähige Stiftungen des öffentlichen Rechts aus (Bundesverband Deutscher Stiftungen 2017, S. 16, 17).

Statistiken, die auf den Informationen aus der Datenbank des Bundesverbandes Deutscher Stiftungen basieren, beziehen sich - wenn nicht anders angegeben auf die für den Wissenschaftlichen Dienst des Bundesverbandes auswertbare Zahl der aktiven rechtsfähigen Stiftungen des bürgerlichen Rechts.

Zwar ist die Zahl der jährlich neu errichteten Stiftungen nach wie vor hoch, jedoch setzt die anhaltende Niedrigzinsphase deutsche Stiftungen zunehmend unter Druck. Wie eine Befragung des StiftungsPanels des Bundesverbandes Deutscher Stiftungen ${ }^{10}$ zeigt, gestaltet sich der reale Kapitalerhalt für viele Stiftungen immer schwieriger: Nach Einschätzung der befragten Stiftungen wird es 2017 nur noch knapp zwei Dritteln von ihnen gelingen, eine Rendite oberhalb der Anfang 2017 prognostizierten Jahresinflationsrate von 1,5 Prozent zu erwirtschaften $(n=255)$.

10 Seit 2013 hat der Bundesverband Deutscher Stiftungen ein StiftungsPanel aufgebaut, mit dem er regelmäßig aktuelle Zahlen zum Stiftungssektor erhebt. Derzeit sind im Panel mehr als 560 Stiftungen registriert. www.stiftungen.org/stiftungspanel 
Gerade kleinere Stiftungen und solche, die nach Beginn der Niedrigzinsphase 2009 gegründet wurden, sind davon betroffen (Bischoff und Ratajszczak 2017, S. 2).

Räumliche Verteilung: Stiftungen sind in Deutschland sehr unterschiedlich verteilt. Die Gründe dafür sind historischer, gesellschaftlicher und ökonomischer Natur. Eine Vielzahl bedeutungsvoller Stiftungen gibt es in den ehemaligen freien Reichs- und Hansestädten mit ihrer bürgerschaftlichen Tradition und einem selbstbewussten Bürgertum, das sich stifterisch in die Gesellschaft einbringt. Die Großstadt mit der höchsten Stiftungsdichte ist Würzburg mit derzeit 91 Stiftungen

Tabelle 3.20 Stiftungsdichte (Stiftungen je 100 ooo Einwohner), -errichtungen, -bestand und -wachstum 2016 (rechtsfähige Stiftungen des bürgerlichen Rechts)

\begin{tabular}{llrrl}
\hline Bundesland & $\begin{array}{l}\text { Stiftungen je } \\
100 \text { 000 EW }\end{array}$ & Errichtungen & Bestand & $\begin{array}{l}\text { Stiftungswachs- } \\
\text { tum im Vergleich } \\
\text { zum Vorjahr in \% }\end{array}$ \\
\hline Hamburg & 78 & 20 & 1393 & 1,3 \\
Bremen & 49 & 7 & 331 & 0,9 \\
Hessen & 32 & 60 & 1970 & 2,8 \\
Bayern & 31 & 100 & 3938 & 2,4 \\
Baden-Württemberg & 30 & 80 & 3255 & 2,1 \\
Niedersachsen & 29 & 54 & 2265 & 2,6 \\
Schleswig-Holstein & 27 & 17 & 761 & 1,1 \\
Berlin & 26 & 29 & 900 & 2,7 \\
Rheinland-Pfalz & 26 & 32 & 1045 & 2,9 \\
Nordrhein-Westfalen & 24 & 134 & 4258 & 2,4 \\
Saarland & 18 & 5 & 177 & 2,9 \\
Thüringen & 15 & 9 & 319 & 2,9 \\
Sachsen & 13 & 16 & 528 & 3,7 \\
Sachsen-Anhalt & 13 & 6 & 289 & 2,5 \\
Mecklenburg-Vorpommern & 10 & 2 & 165 & 0,0 \\
Brandenburg & 9 & 582 & 212 & 5,5 \\
\hline Bundesweit & 27 & & 2,4 \\
\hline
\end{tabular}

Quelle: Umfrage unter den Stiftungsaufsichtsbehörden, Stichtag 31. Dezember 2016. DESTATIS, Fortschreibung des Bevölkerungsstandes, Stichtag 31. Dezember 2015. 
pro 100 ooo Einwohner, gefolgt von Oldenburg mit 80 und Hamburg mit 78 Stiftungen pro 100 ooo Einwohner (Bundesverband Deutscher Stiftungen 2017, S. 26).

Die Zahl der Stiftungen in den alten Bundesländern unterscheidet sich von der in den neuen Bundesländern. Auch wenn prozentual die Zahl der Stiftungen in Brandenburg und Sachsen stärker wächst als im Rest des Landes: In absoluten Zahlen gibt es in den neuen Bundesländern weit weniger Stiftungen (Tab. 3.20). Konnte in der Nachkriegszeit das Stiftungswesen in der Bundesrepublik gedeihen, weil zunehmender Wohlstand und wachsende Privatvermögen einen guten Nährboden schufen, markierte die DDR-Zeit für Stiftungen einen tiefen Einschnitt. Hier wurden viele Stiftungen zusammengelegt oder aufgelöst. Dass auch nach der deutschen Einigung die Zahl der Stiftungserrichtungen im Osten Deutschlands bei Weitem nicht an die in den westlichen Bundesländern heranreicht, liegt unter anderem an der Vermögensverteilung in Deutschland: Immer noch sind die Vermögen der Menschen in den östlichen Ländern zum Teil wesentlich geringer als in den westlichen. Daher steht weniger Geld zum Stiften zur Verfügung.

Wofür Stiftungen sich engagieren: Stiftungen engagieren sich vielfältig. Sie erfüllen ihren Stiftungszweck entweder, indem sie selbst Projekte verwirklichen (operativ), oder indem sie andere Institutionen, Initiativen oder Personen unterstützen (fördernd). Aktuell arbeiten 18 Prozent der rechtsfähigen Stiftungen des bürgerlichen Rechts operativ, 60 Prozent fördernd und 21 Prozent sind sowohl fördernd als auch operativ tätig (Bundesverband Deutscher Stiftungen 2017, S. 35).

Der Stiftungszweck ist ein wesentliches Merkmal einer Stiftung. Stiftende legen die Zwecke, die sie verfolgen möchten, in der Stiftungssatzung fest. Gemeinsam ist den meisten Stiftungen, dass sie einen Bereich gesellschaftlicher Aufgaben fördern, der in den Augen der Stiftenden nicht ausreichend berücksichtigt wird. Dabei sehen sie sich nicht als Ausfallbürgen für Leistungen des Staates.

Mehr als ein Drittel der rechtsfähigen Stiftungen fördert Bildungschancen und Bildungsprojekte in Schulen, Kindergärten oder Universitäten. Fast 30 Prozent der Stiftungen haben Kunst und Kultur in ihrer Satzung verankert (Abb. 3.6). Oft spiegeln sich in der Entwicklung der Stiftungszwecke über die letzten Jahrzehnte auch gesellschaftliche Debatten und Problemlagen wider. So waren zwischen 1951 bis 1960 lediglich 7 Prozent aller Stiftungen im Umweltschutz aktiv. In den letzten Jahren haben die Umweltbewegung sowie Kernkraftkatastrophen wie in Tschernobyl oder Fukushima das grüne Stiftungswachstum angeregt (Bundesverband Deutscher Stiftungen 2017, S. 36). 
Abbildung 3.6 Die 15 häufigsten steuerbegünstigten Stiftungszwecke laut Abgabenordnung (in Prozent)

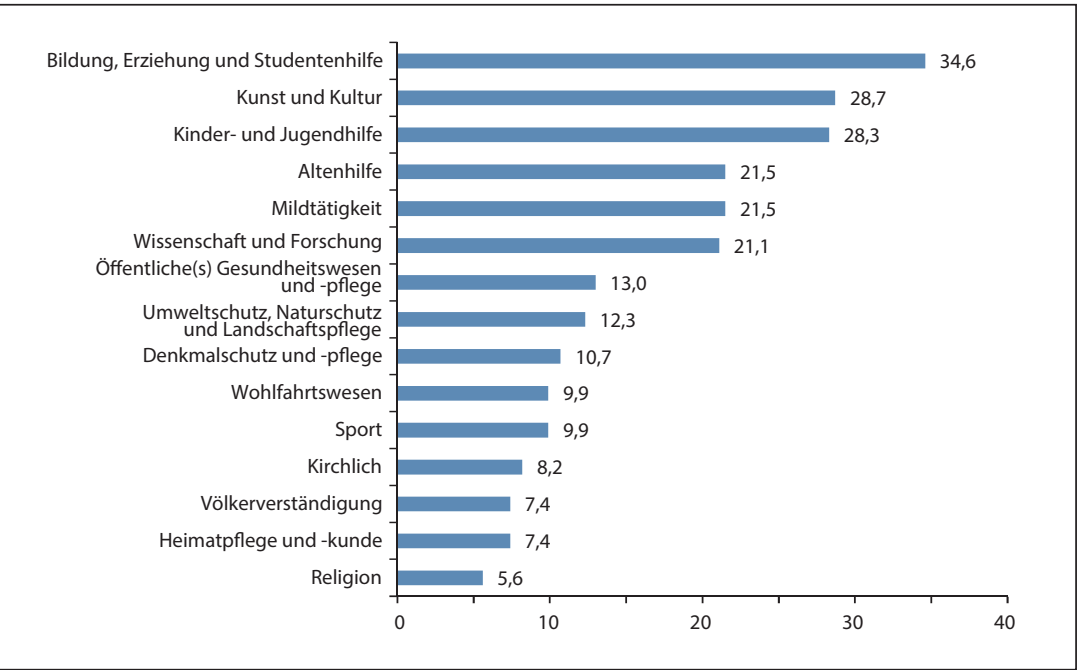

Mehrfachnennungen möglich $(n=19592)$.

Quelle: Datenbank Deutscher Stiftungen, Stand Mai 2017.

Personelle Strukturen: Die arbeitsmarktpolitische Relevanz des Stiftungssektors wächst vermutlich mit der Zahl der Stiftungen. Gleichwohl steckt nicht hinter jeder Stiftung ein Arbeitsplatz. Wie sieht also der „Arbeitsmarkt Stiftungen“ heute aus? Dazu gibt es vergleichsweise wenige Daten. Dass Personalfragen für den strategischen Erfolg von Stiftungen bedeutsam sind, steht sicherlich außer Frage. Allerdings verfügten noch vor einigen Jahren laut einer Befragung des StiftungsPanels nur knapp 16 Prozent der Stiftungen über eine schriftlich festgehaltene Personalstrategie (Bischoff und Hagedorn 2014, S. 3). Eine Befragung des StiftungsPanels aus dem Sommer 2017 zum Thema „Personal in Stiftungen“ zeigt: Gut jede vierte Stiftung besteht nur aus dem Vorstand (Ratajszczak und Bischoff 2017, S. 4). Rund ein Fünftel der befragten Stiftungen hat eine hauptamtliche Geschäftsführung, knapp 30 Prozent haben hauptamtlich Beschäftigte (Abb. 3.7). Die Anzahl der Stellen ist jedoch überschaubar: Mehr als die Hälfte der Stiftungen mit hauptamtlichen Mitarbeitenden hat nur ein bis fünf Angestellte (55,9 Prozent, $\mathrm{n}=68$ ) (ebd., 4). Die meisten Stiftungen sind also Kleinstorganisationen. Zu einem ähnlichen Ergebnis kommt Sandberg (Sandberg 2014, S. 18). 
Abbildung 3.7 Weitere Mitarbeitende neben den Mitgliedern des vertretungsberechtigten Gremiums (in Prozent)

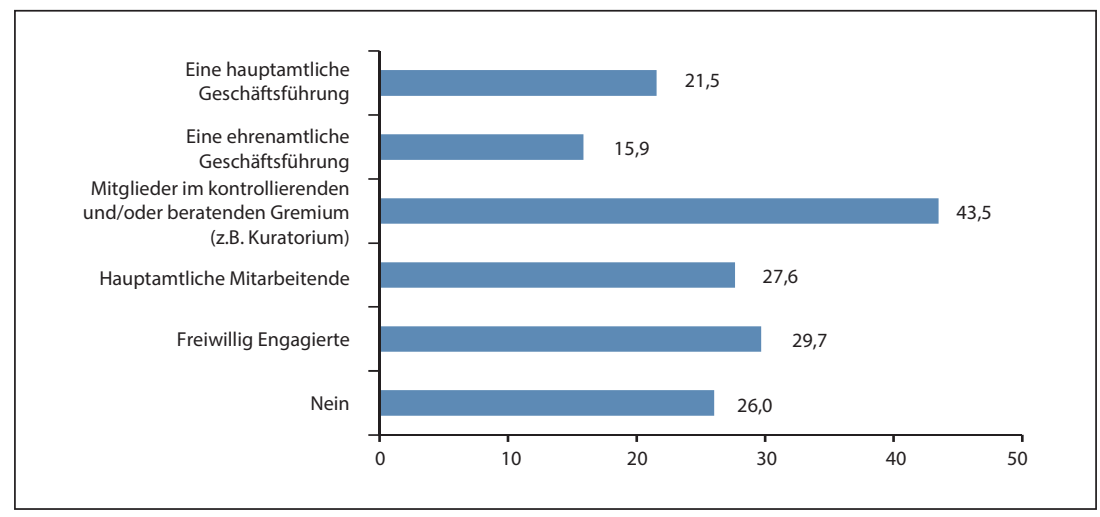

Mehrfachnennungen möglich $(n=246)$.

Quelle: Stiftungsfokus Nr. 13, hg. v. Bundesverband Deutscher Stiftungen. Berlin 2017.

Abbildung 3.8 Beschäftigungsverhältnisse der hauptamtlichen Mitarbeitenden (in Prozent)

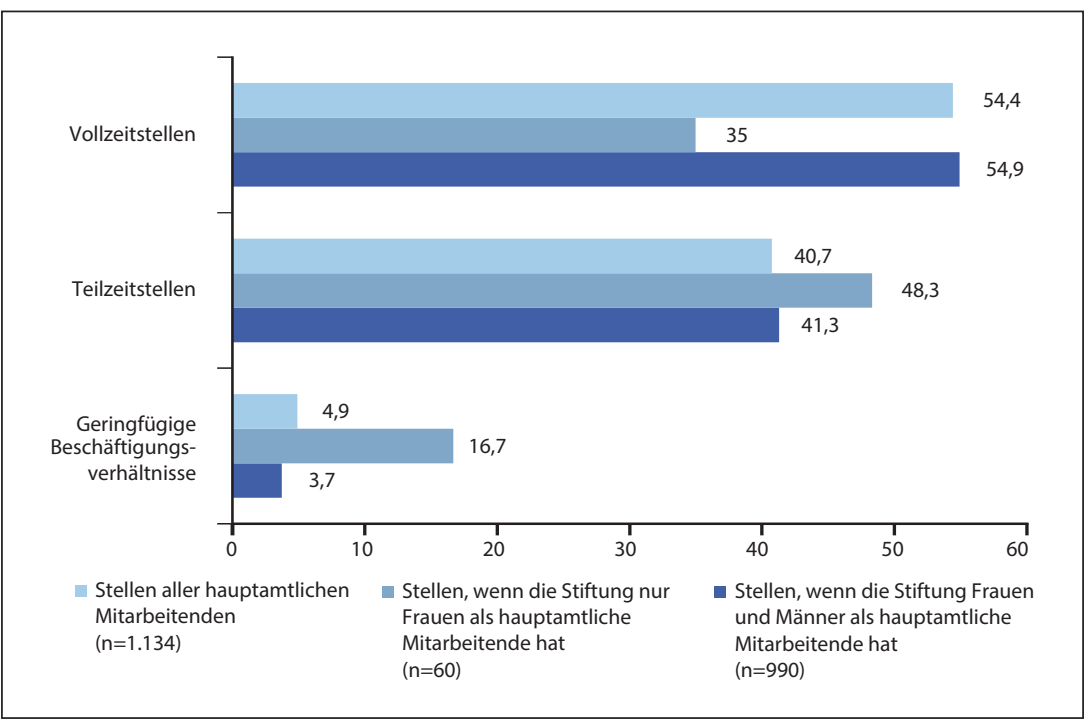

Quelle: Stiftungsfokus Nr. 13, hg. v. Bundesverband Deutscher Stiftungen. Berlin 2017. 
Beschäftigen die befragten Stiftungen hauptamtliche Mitarbeitende, handelt es sich bei mehr als der Hälfte der Arbeitsplätze um Vollzeitstellen (54,4 Prozent), rund 40 Prozent sind Teilzeitstellen (40,7 Prozent) und knapp 5 Prozent geringfügige Beschäftigungsverhältnisse (4,9 Prozent). Im Schnitt (Median) bieten diese Stiftungen vier Vollzeit-, drei Teilzeitstellen und zwei geringfügige Beschäftigungsverhältnisse an. Sind in den befragten Stiftungen nur Frauen als hauptamtliche Mitarbeiterinnen tätig, sinkt der Anteil der Vollzeitstellen. Teilzeitstellen und geringfügige Beschäftigungsverhältnisse nehmen dagegen deutlich zu (Abb. 3.8).

Freiwilliges Engagement: Freiwillige sind für viele Stiftungen von großer Bedeutung: Rund 30 Prozent der mit dem StiftungsPanel befragten Stiftungen arbeiten mit freiwillig Engagierten zusammen (s. Abb. 3.7). Dennoch stellt sich die Frage: Weshalb arbeiten Stiftungen mit Freiwilligen zusammen? Liegt es daran, dass es viele kleine Stiftungen gibt, die sich hauptamtliches Personal nicht leisten können? Oder gibt es auch Stiftungen, die sich ganz bewusst für die Zusammenarbeit mit Freiwilligen entscheiden? Eine Studie des Bundesverbandes Deutscher Stiftungen hat diese Fragen aufgegriffen und bietet einen guten Überblick über das Thema (Bischoff; Hagedorn; Roscher 2015). Einige der dort untersuchten Aspekte wurden im Rahmen der Erhebung zum Personal in Stiftungen über das StiftungsPanel erneut thematisiert bzw. als abgeleitete Frage gestellt: Eine Mehrheit der befragten Stiftungen arbeitet mit Freiwilligen, weil sich dadurch die Qualität ihrer Arbeit

Abbildung 3.9 Gründe für die Zusammenarbeit mit Freiwilligen (in Prozent)

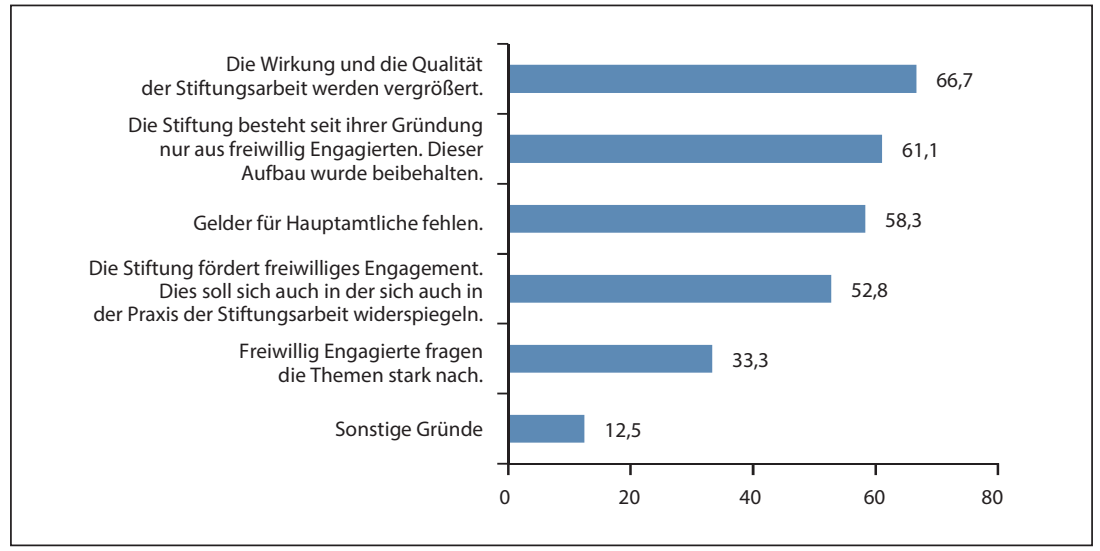

Mehrfachnennungen möglich $(n=72)$.

Quelle: StiftungsPanel-Befragung „Personal in Stiftungen“, Juli 2017. 
erhöht (66,7 Prozent). Knapp 6o Prozent der Befragten haben außerdem angegeben, dass ihnen die finanziellen Mittel für Hauptamtliche fehlen (58,3 Prozent) (Abb. 3.9). Wer Menschen zum Engagement bewegen möchte, kommt um die direkte Ansprache nicht herum: Über 8o Prozent der befragten Stiftungen gewinnen auf diese Weise Freiwillige (84,7 Prozent) (Ratajszczak und Bischoff 2018, S. 6). ${ }^{11}$

Stiftungskooperationen: Häufig ist zu hören, dass Stiftungen in Zeiten geringer Zinserträge vermehrt auf Kooperationen setzen (sollten). Bei einer Befragung des StiftungsPanels Ende 2014 zu Kooperationen hat fast die Hälfte der Stiftungen als Grund für die Kooperation „Finanzielle Unterstützung“ genannt (49,4 Prozent, $\mathrm{n}=87$ ) (Hagedorn; Bischoff 2015, S. 6). Allerdings können die Ergebnisse dieser StiftungsPanel-Befragung nicht direkt in Bezug zur Niedrigzinsphase gesetzt werden. Eine aktuelle Befragung des StiftungsPanels von Anfang 2017 zeigt: Nur ein knappes Drittel der Stiftungen ist 2016 Kooperationen eingegangen (31,8 Prozent, $\mathrm{n}=255$ ), dagegen war dies laut Umfrage 2014 bei über der Hälfte der Teilnehmenden der Fall (51,5 Prozent, $\mathrm{n}=169$ ) (Hagedorn und Bischoff 2015, S. 3). Aktuell kooperieren kleine Stiftungen mit einem Stiftungskapital bis unter einer Million Euro deutlich seltener als große ab einer Million Euro Kapital (24,2 Prozent vs. 38,5 Prozent) (Abb. 3.10).

Die drei häufigsten Kooperationspartner der Befragten sind derzeit (Mehrfachnennungen möglich): andere Stiftungen mit 75,3 Prozent, Vereine und Verbände mit 46,9 Prozent sowie Universitäten mit 30,9 Prozent (Abb. 3.11). Die Reihenfolge der ersten drei Nennungen ist gegenüber 2014 gleich geblieben, lediglich die Anteile haben sich leicht verändert (Bischoff und Ratajszczak 2017, S. 10). Niedrige Zinsen führen im Übrigen nicht unbedingt zu veränderten Kooperationsstrategien, denn nur eine Minderheit der befragten Stiftungen plant, wegen der anhaltenden Niedrigzinsphase verstärkt zu kooperieren $(27,1$ Prozent, $\mathrm{n}=255) .{ }^{12}$

11 In der Befragung zum „Personal in Stiftungen“ vom Juli 2017 wurden auch Daten zu den freiwillig Engagierten in den Stiftungen erhoben.

12 Ebd., S. 11, Werte „trifft voll zu“ und „trifft eher zu“ auf einer 5er-Skala der Frage „Die anhaltende Niedrigzinsphase wird dazu führen, dass wir künftig verstärkt auf Kooperationen setzen“. 
Abbildung 3.10 Kooperationen von Stiftungen (in Prozent): Nur knapp ein Drittel der Stiftungen hat 2016 mit anderen kooperiert

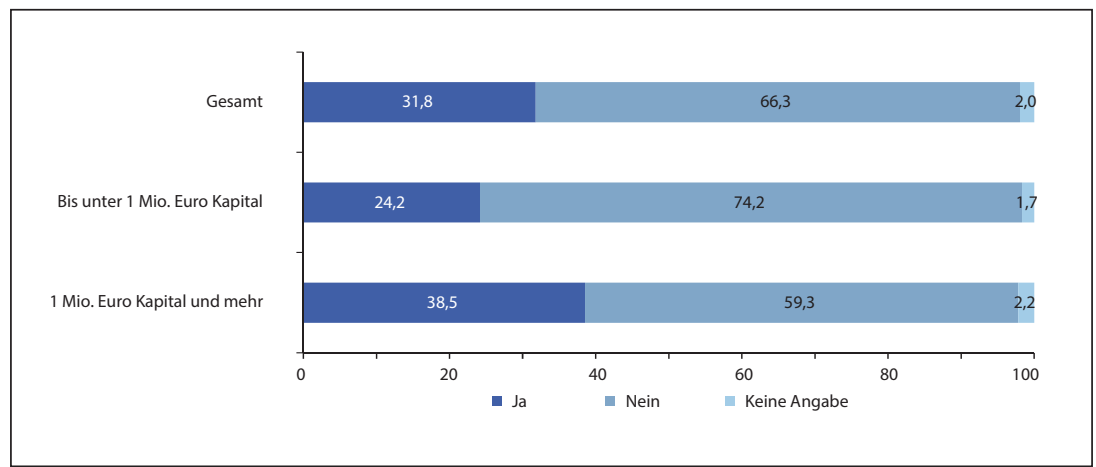

Quelle: Stiftungsfokus Nr. 11, hg. v. Bundesverband Deutscher Stiftungen. Berlin 2017.

\section{Abbildung 3.11 Kooperationspartner von Stiftungen (in Prozent)}

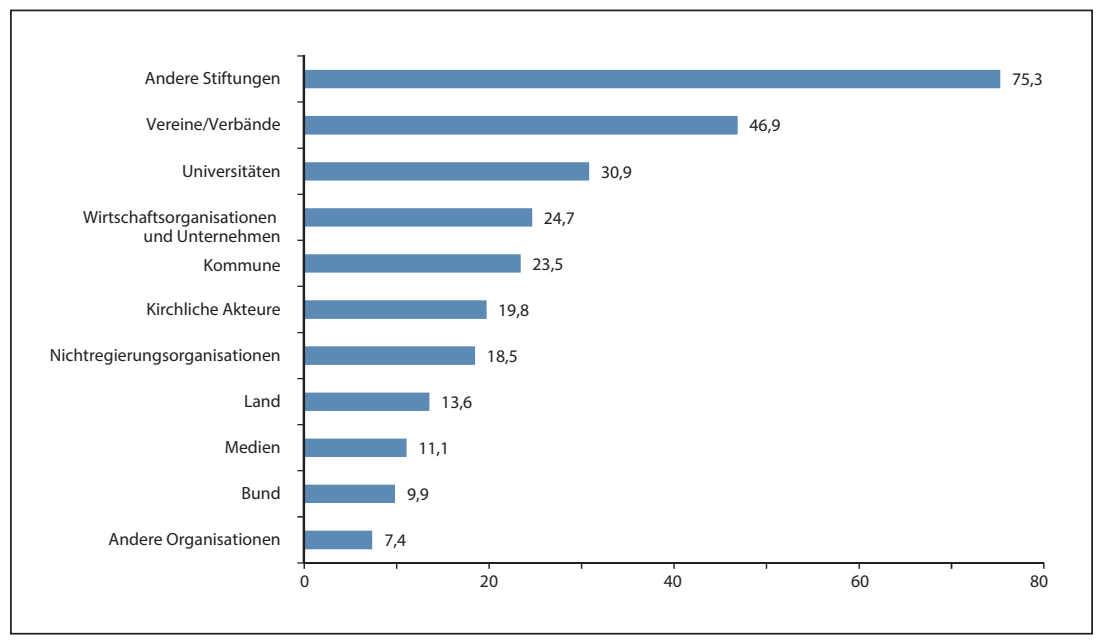

Mehrfachnennungen möglich $(n=81)$.

Quelle: Stiftungsfokus Nr. 11, hg. v. Bundesverband Deutscher Stiftungen. Berlin 2017. 


\subsection{Wirtschaftlich Aktive ${ }^{13}$}

Im Jahr 2014 waren im Dritten Sektor laut statistischem Unternehmensregister mehr als drei Millionen Personen sozialversicherungspflichtig beschäftigt (vgl. Kapitel 5) ${ }^{14}$, also dreimal so viele wie in Banken, Versicherungen und anderen zum finanziellen Sektor zählenden Institutionen zusammen angestellt sind. Jeder zehnte sozialversicherungspflichtig Beschäftigte in Deutschland arbeitet somit in einer Organisation oder Einrichtung des Dritten Sektors. Allein die gemeinnützigen Unternehmen im Gesundheits- und Sozialwesen stellen knapp zwei Millionen sozialversicherungspflichtige Arbeitsplätze. Hinzu kommen über alle Wirtschaftsbereiche verteilt noch rund 350 ooo geringfügig Beschäftigte.

Die zur Zivilgesellschaft zählenden wirtschaftlich aktiven Organisationen sind vor allem in den in Abbildung 3.12 aufgezeigten fünf Wirtschaftsbereichen tätig. Nahezu 9o Prozent der zum Wirtschaftszweig „Interessenvertretungen, religiöse Vereinigungen“ gehörenden institutionellen Einheiten sind dem Dritten Sektor und damit der Zivilgesellschaft zuzuordnen. Dazu zählen alle religiösen Vereinigungen, Gewerkschaften, Verbraucherverbände, Parteien und andere nicht unmittelbar für Unternehmen tätige Organisationen. Berufsorganisationen wie Ärzte- oder Rechtsanwaltsvereinigungen wurden nicht zum Dritten Sektor gezählt.

Auch im Bereich „Erziehung und Unterricht", der ansonsten überwiegend staatlich geprägt ist, kommt Einrichtungen in der Trägerschaft von zivilgesellschaftlichen Organisationen mit anteilig knapp einem Viertel aller Organisationen eine große Bedeutung zu. Hierzu zählen beispielsweise Kindergärten und Schulen in kirchlicher oder freigemeinnütziger Trägerschaft. Daneben sind zivilgesellschaftliche Organisationen stark in den Bereichen „Kunst, Unterhaltung und Erholung“ (rund 15 Prozent aller dort zugeordneten Einheiten), „Gesundheits- und Sozialwesen“ (1o Prozent aller Einheiten) sowie im Bereich „Forschung und Entwicklung“ (rund 8 Prozent aller Einheiten) tätig. Zum Dritten Sektor zählen neben Vereinen, Stiftungen und Religionsgemeinschaften auch gemeinnützige Genossenschaften oder gemeinnützige Gesellschaften mit beschränkter Haftung $(\mathrm{GmbH})$. Diese zivilgesellschaftliche Organisationen ergänzen die von staatlichen oder auch marktwirtschaftlich tätigen Unternehmen gemachten Angebote, beispielsweise indem sie Pflege- und Altenheime oder auch Krankenhäuser führen sowie kulturelle Angebote wie Theater, Kino oder Museen bereitstellen.

13 Dieses Unterkapitel wurde verfasst von Jana Priemer, ZiviZ und Christian Hohendanner, IAB.

14 Hierzu zählen auch sozialversicherungspflichtig beschäftigte Menschen mit Behinderungen. 
Abbildung 3.12 Anteil der Unternehmen/Vereine des Dritten Sektors an allen wirtschaftlich aktiven Einheiten im jeweiligen Wirtschaftsbereich (in Prozent)

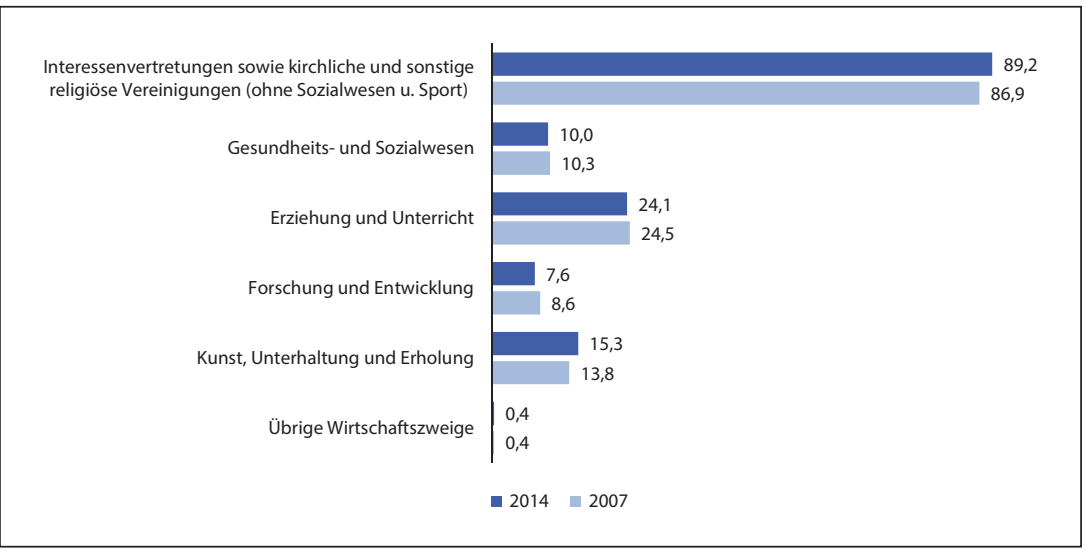

Quelle: Statistisches Unternehmensregister des Statistischen Bundesamtes.

\subsection{Wohlfahrtspflege - zwischen Staat, Markt und Gemeinwohl ${ }^{15}$}

Die Spitzenverbände der Bundesarbeitsgemeinschaft der Freien Wohlfahrtspflege (BAGFW) führen alle vier Jahre die Daten der jeweiligen Verbände zu einer Gesamtstatistik zusammen. Die letzte verfügbare Gesamtstatistik der Einrichtungen und Dienste der Freien Wohlfahrtspflege bezieht sich auf das Jahr 2012. Erfasst wurden die Angebote als institutionalisierte Leistungen der Rechtsträger, Mitgliedsorganisationen der Spitzenverbände der Freien Wohlfahrtspflege, die diese Leistungen zur Deckung des Bedarfs von hilfebedürftigen Personen bereitstellen. Die Angebote sind nicht mit örtlichen Einheiten (örtlich getrennte Betriebsstätten, Standorte oder Niederlassungen) eines Rechtsträgers gleichzusetzen. Mehrere Angebote eines Rechtsträgers können daher an einem Standort betrieben werden. Ein Beispiel ist das Evangelische Johannesstift Berlin, das sowohl Krankenhäuser, Angebote aus den Bereichen Alten-, Behinderten- und Kinder- und Jugendhilfe betreibt als auch Bildungseinrichtungen und Integrationsunternehmen anbietet.

Im Jahr 2012 wurden in der Freien Wohlfahrtspflege bundesweit 140764 Angebote und Dienste mit über drei Millionen Betten bzw. Plätzen erfasst (BAGFW

15 Dieses Unterkapitel wurde verfasst von Wolfgang Schmitt, BAGFW. 
Abbildung 3.13 Entwicklung der Angebote der Freien Wohlfahrtspflege in den Jahren 2004 bis 2012

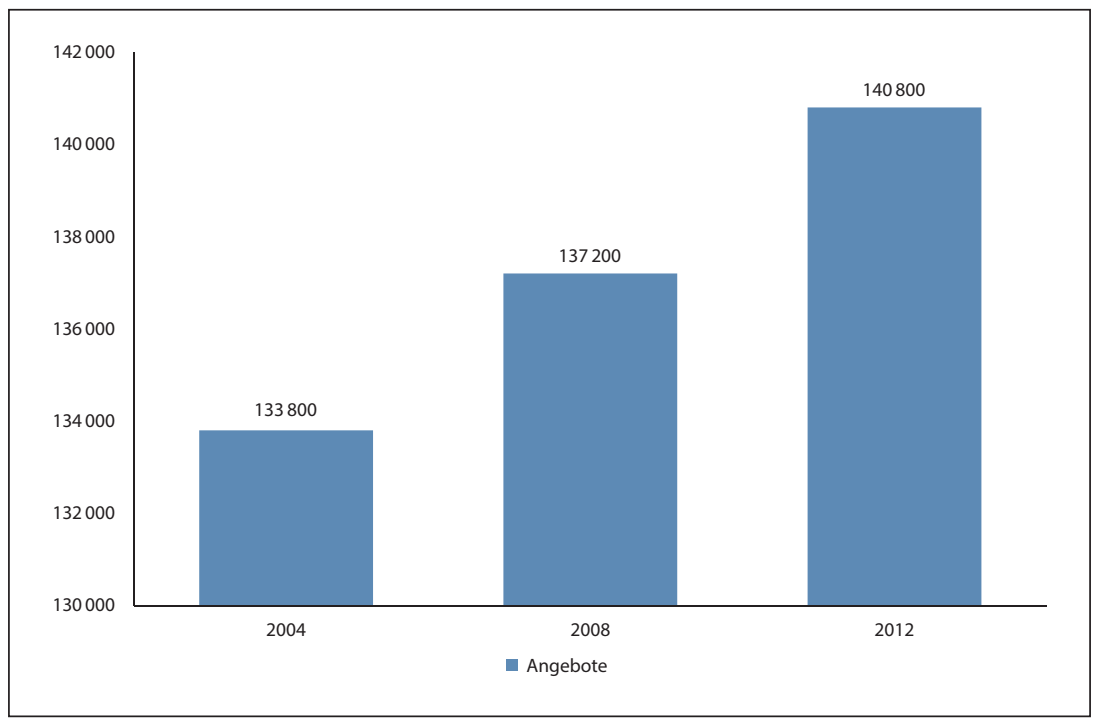

Quelle: Bundesarbeitsgemeinschaft der Freien Wohlfahrtspflege e.V.(BAGFW) (2014).

2014). Davon fallen 35469 Angebote auf den Bereich der Selbsthilfe- und Helfergruppen und der Gruppen des bürgerschaftlichen Engagements. Helfergruppen der Krankenhaus- und Altenheimhilfe, Hausaufgabenhilfegruppen und andere Schülerhilfegruppen, Familien- und Nachbarschaftszentren, Generationenhäuser, Laienmitarbeitergruppen für psychisch kranke und behinderte Menschen, Telefondienste und Telefonketten sind beispielhafte Angebote in diesem Bereich. Nicht enthalten sind zusätzliche, zeitlich befristete Maßnahmen sowie Aktivitäten der Auslands- und Katastrophenhilfe. Damit hat sich die Zahl der Angebote und Dienste gegenüber dem Jahr 2004 von 133760 auf 140764 bzw. um 5 Prozent erhöht.

Die größten Zuwachsraten bei der Anzahl der Angebote und Dienste sind in den weiteren Hilfen (23 Prozent) und den Hilfen für Personen in besonderen sozialen Situationen (22 Prozent) zu verzeichnen. $\mathrm{Zu}$ den weiteren Hilfen zählen unter anderem Wohnheime für Studierende, Wohnheime an Ausbildungsstätten für soziale Berufe, allgemeine Sozialberatungsstellen, Möbellager und Geschäftsstellen; zu den Hilfen für Personen in besonderen sozialen Situationen gehören unter anderem Übernachtungswohnheime und Notunterkünfte, Nachsorgeeinrichtun- 
Tabelle 3.21 Angebote und Dienste der Freien Wohlfahrtspflege nach Arbeitsfeldern in den Jahren 2004, 2008 und 2012

\begin{tabular}{lrrr}
\hline Angebote & 2004 & 2008 & \multicolumn{1}{c}{2012} \\
\hline Gesundheitshilfe & 7900 & 8500 & 7500 \\
Jugendhilfe & 36400 & 38100 & 38400 \\
Familienhilfe & 7600 & 7200 & 4600 \\
Altenhilfe & 15800 & 16500 & 18100 \\
Behindertenhilfe & 14300 & 15400 & 16400 \\
$\begin{array}{l}\text { Hilfe für Personen in besonderen sozialen Situa- } \\
\text { tionen }\end{array}$ & 7200 & 7800 & 8800 \\
$\begin{array}{l}\text { Weitere Hilfen } \\
\begin{array}{l}\text { Aus-, Fort- und Weiterbildungsstätten für soziale } \\
\text { und pflegerische Berufe }\end{array}\end{array}$ & 15000 & 7300 & 9900 \\
$\begin{array}{l}\text { Selbsthilfegruppen und Gruppen des bürger- } \\
\text { schaftlichen Engagements }\end{array}$ & 34900 & 34800 & 1600 \\
\hline
\end{tabular}

Quelle: Bundesarbeitsgemeinschaft der Freien Wohlfahrtspflege e.V.(BAGFW) (2014).

gen für Menschen mit Abhängigkeitserkrankungen, Tagesstätten/Zentren für MigrantInnen und Angehörige, Beschäftigungs- und Qualifizierungseinrichtungen beziehungsweise Projekte für erwerbslose Menschen, Schuldnerberatungsstellen oder Telefonseelsorgestellen. Durchweg positiv sind die Wachstumsraten bei den Angeboten und Diensten der Behindertenhilfe (15 Prozent) und der Altenhilfe (14 Prozent). Mit einem Anstieg von 5 Prozent ist auch die Jugendhilfe ein Wachstumsbereich in der Freien Wohlfahrtspflege. In der Familienhilfe ist seit dem Jahr 2004 ein Rückgang der Einrichtungen und Dienste um 40 Prozent zu beobachten, der zu einem Großteil mit Verschiebungen der Erhebungskategorien in andere Arbeitsfelder zusammenhängt. So wurden zum Beispiel die Sozialstationen der Altenhilfe zugeordnet. Im Bereich der Gesundheitshilfe gibt es einen Rückgang der Angebote um 5 Prozent. Die Zahl der Selbsthilfegruppen und Gruppen des bürgerschaftlichen Engagements in der Freien Wohlfahrtspflege nahm insgesamt um 2 Prozent zu. Größter Arbeitsbereich der Freien Wohlfahrtspflege in Bezug auf die Angebote und Dienste ist die Jugendhilfe mit 38367 Einrichtungen. Das entspricht einem Anteil von über einem Drittel (36 Prozent) aller Angebote. Danach folgen die Altenhilfe und die Behindertenhilfe, die mit 18051 bzw. 16446 Angeboten und Diensten einen Anteil von 17 bzw. 16 Prozent ausmachen. 
Abbildung 3.14 Angebote und Dienste der Freien Wohlfahrtspflege nach Arbeitsfeldern im Jahr 2012 (in Prozent)

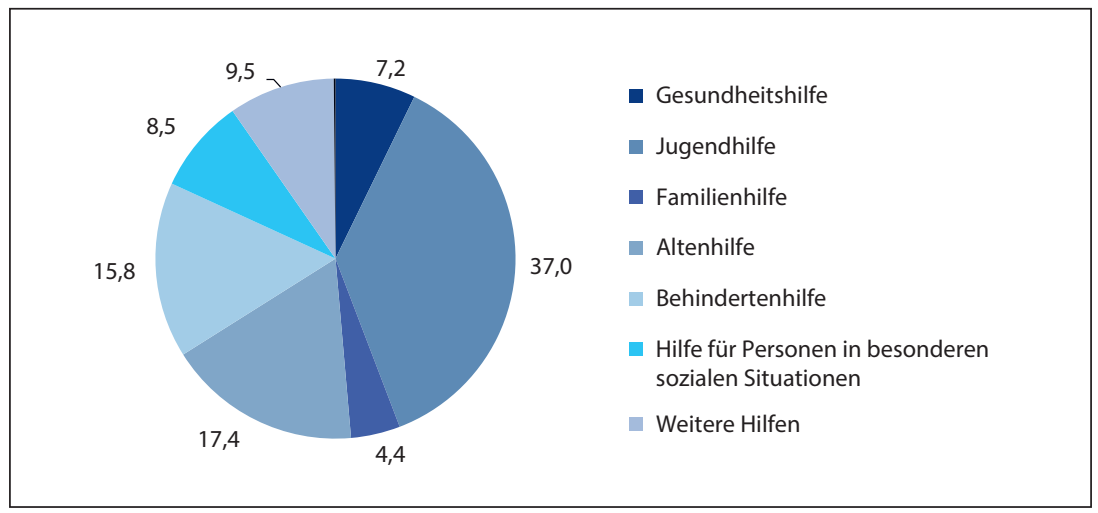

Quelle: Bundesarbeitsgemeinschaft der Freien Wohlfahrtspflege e.V.(BAGFW) (2014).

\subsection{Kontextualisierung und Fazit}

Anhand der vorliegenden Daten können einige aktuelle Entwicklungen in der organisierten Zivilgesellschaft dargestellt werden, aus denen sich Schlüsse für die engagementpolitische Praxis sowie für organisationsinterne Entwicklungsprozesse ziehen lassen.

Die Ergebnisse verdeutlichen die Vielfalt in der Organisationslandschaft und verweisen zugleich auf die hohe gesellschaftliche Relevanz zivilgesellschaftlicher Organisationen. Der eingetragene Verein ist nach wie vor ein Erfolgsmodell: Noch immer werden mehr neue Vereine gegründet als gelöscht; die Mitgliederzahlen sind bei den meisten stabil. Ernstzunehmende Rückgänge sind bislang vor allem im ländlichen Raum zu beobachten. Auch das Stiftungswachstum hält an; weiterhin werden neue Stiftungen errichtet. Die Daten zeigen auch, dass es parallel eine weitere Entwicklung gibt: Neben den meist ehrenamtlich getragenen Organisationen hat sich ein professionell aufgestelltes Segment von Organisationen entwickelt, dessen Bedeutung in den vergangenen Jahren rasant zugenommen hat. In einem Zeitraum von nur neun Jahren ist die Zahl der eingetragenen gemeinnützigen $\mathrm{GmbHs}$ von gut 16 ooo auf etwa 25000 angestiegen. Jeder zehnte sozialversicherungspflichtig Beschäftigte ist heute in einer Organisation der Zivilgesellschaft angestellt. Viele von ihnen tragen zur sozialen Wohlfahrtspflege bei, wie das Beispiel der Wohlfahrtsverbände zeigt. 
Auch wenn ein Teil der Organisationen inzwischen mit bezahlten Beschäftigten arbeitet und die Wirtschaftskraft dieser Organisationen unbestritten ist, sind die meisten Vereine und auch Stiftungen kleine Organisationen. Sie werden häufig von bürgerschaftlichem Engagement getragen und sind daher in höchstem Maße von diesem abhängig. Die Mobilisierung von Engagierten für einen langfristigen Einsatz wurde als einer der größten Problembereiche ausgemacht, obgleich die meisten Organisationen ihre Engagiertenzahlen stabil halten konnten. Vielen Organisationen fällt es immer schwerer, Engagierte dauerhaft zu binden. Vereine im ländlichen Raum stellt dies vor besonders ernst zu nehmende Probleme. In manchen von Abwanderung betroffenen Kommunen bieten Vereine die letzten verbliebenen öffentlichen Infrastrukturen, so dass ihnen dort eine herausragende Funktion für die gesellschaftliche Teilhabe zukommt. Wie der Dorfverein überleben kann, sollte daher auch zukünftig eine der zentralen engagementpolitischen Fragen sein. Auch die generelle Stärkung des ehrenamtlichen und freiwilligen Engagements als zentrale Ressource der Vereinsarbeit muss weiterhin im Fokus der Überlegungen stehen. Hierbei sind auch die Organisationen selbst gefragt, die sich überlegen müssen, wie sie attraktive Engagementgelegenheiten anbieten können.

Bereits diese kurz angerissenen Problemlagen verdeutlichen, dass Daten zur organisierten Zivilgesellschaft unterschiedliche Anforderungen erfüllen müssen. Sie sollten allgemeine Trends aufzeigen können, um bereichsübergreifende Betrachtungen zu ermöglichen und etwaige Verschiebungen innerhalb des Sektors aufzuzeigen. Ein für den Sport typischer Befund muss auf andere Bereiche längst nicht zutreffen. Gleichzeitig sollten Daten dazu beitragen, dass bestimmte Entwicklungen nicht aus dem Raster der Wahrnehmung fallen. Dass bildungsbezogenes Engagement heute zu einem der beliebtesten Engagementfelder zählt, zeigen sowohl die Daten des Freiwilligensurveys und des ZiviZ-Surveys als auch die des Bundesverbandes Deutscher Stiftungen. Dennoch ist Engagement im Bildungskontext heute noch immer ein vernachlässigtes Forschungsfeld, für das es kaum bereichsspezifische Daten gibt.

Damit zeigt sich eine weitere Anforderung an Daten zur organisierten Zivilgesellschaft: Neben der Abbildung der Makroperspektive braucht es zusätzliche Daten, die Einblicke in die Tiefe einzelner Teilbereiche ermöglichen. Nur so lassen sich bereichsspezifische Fragestellungen beantworten. Die Daten der Sportentwicklungsberichte liefern beispielsweise spezifische Erkenntnisse über die Entwicklungen, Herausforderungen und Probleme der über 90000 gemeinnützig und verbandlich (im DOSB) organisierten Sportvereine in Deutschland. Sie sind daher ein zentrales Instrument für eine datenbasierte Sportpolitikberatung, aus dem sich Argumentations- und Handlungswissen für die Mitgliedsorganisationen im DOSB ableiten lassen. Ein Instrument wie das der Sportentwicklungs- 
berichte wäre daher auch für andere Teilbereiche wünschenswert. Vor allem für Organisationszweige mit vielen jungen Organisationen, die in den vergangenen Jahren besonders viel Zulauf erhalten haben (wie Bürger- und Verbraucherinteressen oder Internationale Solidarität) sollten solche datenbasierten Instrumente stärker in den Fokus gerückt werden. Die Erkenntnisse und das daraus abgeleitete Handlungswissen dürften auch für jene Organisationen gewinnbringend sein, bei denen die Gewinnung und Bindung von neuen Mitgliedern nicht (mehr) allzu positiv ist.

Ein Blick in die Tiefe fehlt bislang auch für wirtschaftlich aktive Organisationen. Die vorgestellten Daten des IAB-Betriebspanels und des Statistischen Bundesamtes zeigen eindrücklich die wachsende Bedeutung von gemeinnützigen $\mathrm{GmbHs}$ und anderen wirtschaftlich, aber gemeinnützig arbeitenden Organisationen. Doch es bleiben Fragen offen, etwa wie stark diese Organisationen von zivilgesellschaftlichem Engagement abhängig sind. Hierzu wäre das Verhältnis von hauptamtlich angestelltem Personal und Ehrenamtlichen ebenso interessant wie ein Blick auf die Finanzierungsstrukturen. Denn wie wir aus dem ZiviZ-Survey wissen (vgl. Kapitel 6), machen Spenden und Mitgliedsbeiträge auch für wirtschaftlich aktive Organisationen einen erheblichen Teil ihrer Finanzierung aus. Über die Zusammenarbeit zwischen Haupt- und Ehrenamt sind zwar Erkenntnisse für die Engagementbereiche Sport, Pflege und Kultur vorhanden (BMFSFJ/ INBAS 2015), aber nicht für die jüngeren Engagementbereiche wie etwa Bildung. Gerade im Bereich Bildung sind vergleichsweise viele Personen angestellt (vgl. Kapitel 5).

Eine weitere Datenlücke besteht auf kommunaler Ebene. Im Datensatz des ZiviZ-Surveys etwa sind 6300 Organisationen enthalten. Mit dieser Anzahl von Datensätzen ist zwar ein Blick in die Tiefe möglich, doch Aussagen über die kommunale Ebene können auf Basis dieser Daten nur in Ausnahmefällen getroffen werden. Dazu bräuchte es weit größere Fallzahlen, wie sie die Sportentwicklungsberichte oder auch die Stiftungsdatenbank vorweisen können.

\subsection{Literatur}

Anheier, H. K., Priller, E., \& Zimmer, A. (200o). Zur zivilgesellschaftlichen Dimension des Dritten Sektors. In H.-D. Klingemann \& F. Neidhardt (Hrsg.) Zur Zukunft Der Demokratie (S. 71-98). Berlin: Sigma.

Bischoff, A., \& Hagedorn, S. (2014). Strategisches Management in Stiftungen. Stiftungsfokus Nr. 2, hg. v. Bundesverband Deutscher Stiftungen. Berlin.

Bischoff, A., Hagedorn, S., \& Roscher, R. (2015). Freiwilliges Engagement in Stiftungen. StiftungsStudie, hg. v. Bundesverband Deutscher Stiftungen. Berlin. 
Bischoff, A., \& Ratajszczak, T. (2017). Stiftungen in der Niedrigzinsphase - aktuelle Zahlen und Fakten. Stiftungsfokus Nr. 11, hg. v. Bundesverband Deutscher Stiftungen. Berlin.

Breuer, C. (2005). Warum Sportvereine überleben - ein managementtheoretisches Gegenmodell zu populären Annahmen. F.I.T. - Das Wissenschaftsmagazin der Deutschen Sporthochschule Köln.

Breuer, C., \& Feiler, S. (2017a). Sportvereine in Deutschland - Ein Überblick. In C. Breuer (Hrsg.), Sportentwicklungsbericht 2015/2016. Band I. Analyse zur Situation der Sportvereine in Deutschland. (S. 15-46). Hellenthal: Sportverlag Strauß.

Breuer, C., \& Feiler, S. (2017b). Sportverein im Wandel - Zur Entwicklung der Sportvereine in Deutschland von 2005 bis 2015. In C. Breuer (Hrsg.), Sportentwicklungsbericht 2015/2016. Band I. Analyse zur Situation der Sportvereine in Deutschland (S. 243-263). Hellenthal: Sportverlag Strauß.

Bundesamt für Justiz (2017). Zusammenstellung der Geschäftsübersichten der Amtsgerichte für die Jahre 1995 bis 2016. Download unter: www.bundesjustizamt.de. Zugegriffen: 28. November 2017.

Bundesarbeitsgemeinschaft der Freien Wohlfahrtspflege e. V.(BAGFW) (2014). Einrichtungen und Dienste der Freien Wohlfahrtspflege, Gesamtstatistik 2012. Download unter: http://www.bagfw.de/fileadmin/user_upload/Broschuere_Gesamt statistik_2012_Webversion.pdf. Zugegriffen: 11. Dezember 2017.

Bundesministerium für Familie, Senioren, Frauen und Jugend (HG) (2017). Engagement in der Flüchtlingshilfe. Ergebnisbericht einer Untersuchung des Instituts für Demoskopie Allensbach. Berlin. Download unter: https://www.bmfsfj.de/ blob/122010/d35ec9bf4a940ea49283485db4625aaf/engagement-in-der-fluech lingshilfe-data.pdf. Zugegriffen: 30. Mai 2018.

Bundesministerium für Familie, Senioren, Frauen und Jugend, INBAS-Sozialforschung $\mathrm{GmbH}$ (HG) (2015). Kooperation von Haupt und Ehrenamtlichen in Pflege, Sport und Kultur. Endbericht. https://www.bmfsfj.de/blob/94178/4c96 c074b83445f80315996abd9c1283/kooperation-von-haupt-und-ehrenamtlichenin-pflege-sport-und-kultur-endbericht-data.pdf. Zugegriffen: 30. Mai 2018.

Bundesverband Deutscher Stiftungen (Hrsg.) (2017). Zahlen, Daten, Fakten zum Deutschen Stiftungswesen. Berlin.

Croon, H. (1959). Niedergang des Vereins. Merkur XIII, S. 262-269.

DOSB (2016). Bestandserhebung 2016. 1. Auflage November 2016. Download unter: www.dosb.de/de/service/download-center/statistiken/. Zugegriffen: 7. Dezember 2017 .

Gemeinsames Registerportal der Länder: https://www.handelsregister.de. Zugegriffen: 25. April 2018.

Hagedorn, S., \& Bischoff, A. (2015). Stiftungskooperationen. Stiftungsfokus Nr. 5, hg. v. Bundesverband Deutscher Stiftungen. Berlin.

Karakayali, S., \& Kleist, O. (2016). EFA-Studie 2, Strukturen und Motive der ehrenamtlichen Flüchtlingsarbeit (EFA) in Deutschland. 2. Forschungsbericht: Ergebnisse einer explorativen Umfrage vom November/Dezember 2015. Download unter: http://www.fluechtlingsrat-brandenburg.de/wp-content/uploads/2016/o8/Stu die_EFA2_BIM_11082016_VOE.pdf. Zugegriffen: 7. Dezember 2017. 
Krimmer, H., \& Priemer, J. (2013). ZiviZ-Survey 2012: Zivilgesellschaft verstehen. Berlin. Download unter: http://www.ziviz.de/publikationen. Zugegriffen: 27. Februar 2018.

Krimmer, H., Priemer, J., \& Labigne, A. (2017). ZiviZ-Survey 2017. Vielfalt verstehen. Zusammenhalt stärken. Berlin. Download unter: http://www.ziviz.de/publika tionen. Zugegriffen: 27. Februar 2018.

Kröhnert, S., Kuhn, E., Karsch, M., \& Klingholz, R. (2011). Die Zukunft der Dörfer. Zwischen Stabilität und Niedergang. Hg. v. Berlin-Institut für Bevölkerung und Entwicklung. Berlin. Online verfügbar unter http://www.berlin-institut.org/filead min/user_upload/Doerfer_2011/Die_Zukunft_der_Doerfer_Webversion.pdf. Zugegriffen: 3. Mai 2017.

Leseberg, N., \& Timmer, K. (2015). Stifterinnen und Stifter in Deutschland. Engagement - Motive - Ansichten, hg. v. Bundesverband Deutscher Stiftungen. Berlin.

Mutz, G., Costa-Schott, R., Hammer, I., Layritz, G., Lexhaller, C., Mayer, M., Poryadina, T., Ragus, S., \& Wolff, L. (2015). Engagement für Flüchtlinge in München. Ergebnisse eines Forschungsprojekts an der Hochschule München in Kooperation mit dem Münchner Forschungsinstitut mis. Online verfügbar unter: http://www. ehrenamtsbibliothek.de/literatur/pdf_1352.pdf. Zugegriffen: 12. Dezember 2017.

Ratajszczak, T., \& Bischoff, A. (2017). Personal in Stiftungen - aktuelle Zahlen und Fakten. Stiftungsfokus Nr. 13, hg. v. Bundesverband Deutscher Stiftungen. Berlin.

Ratajszczak, T., \& Bischoff, A. (2018). Freiwilliges Engagement trägt Stiftungsarbeit. Aktuelle Zahlen und Fakten. Stiftungsfokus Nr. 16, hg. vom Bundesverband Deutscher Stiftungen. Berlin.

Roth, R. (2003). Die dunklen Seiten der Zivilgesellschaft: Grenzen einer zivilgesellschaftlichen Fundierung von Demokratie. Forschungsjournal Neue Soziale Bewegungen, Jg. 16 (2003), H. 2, S. 59-73.

Sandberg, B. (2014). Einführung in den Arbeitsmarkt Stiftungswesen. In B. Sandberg \& C. Mecking. (Hrsg.), Arbeitsplatz Stiftungen. Karrierewege im Stiftungsmanagement. Personalmanagement in Stiftungen, Bd. 3. Essen.

Skurnog, M. (2017). Begriffsschärfung. Bereiche. Besonderheiten. Informelles Engagement. Expertise für das Projekt Menschen.Leben.Integration. Berlin. Download unter: http://www.ziviz.de/publikationen. Zugegriffen: 27. Februar 2018.

Open Access Dieses Kapitel wird unter der Creative Commons Namensnennung 4.0 International Lizenz (http://creativecommons.org/licenses/by/4.0/deed.de) veröffentlicht, welche die Nutzung, Vervielfältigung, Bearbeitung, Verbreitung und Wiedergabe in jeglichem Medium und Format erlaubt, sofern Sie den/die ursprünglichen Autor(en) und die Quelle ordnungsgemäß nennen, einen Link zur Creative Commons Lizenz beifügen und angeben, ob Änderungen vorgenommen wurden.

Die in diesem Kapitel enthaltenen Bilder und sonstiges Drittmaterial unterliegen ebenfalls der genannten Creative Commons Lizenz, sofern sich aus der Abbildungslegende nichts anderes ergibt. Sofern das betreffende Material nicht unter der genannten Creative Commons Lizenz steht und die betreffende Handlung nicht nach gesetzlichen Vorschriften erlaubt ist, ist für die oben aufgeführten Weiterverwendungen des Materials die Einwilligung des jeweiligen Rechteinhabers einzuholen.



